\title{
ANÁlisis FLORÍSTICO DE UN BOSQUE DE ABIES Y EL BOSQUE MESÓFILO DE MONTAÑA ADYACENTE EN JUANACATLÁN, Mascota, Jalisco, MÉXICO
}

\author{
Ricardo Guerrero-Hernández, Jesús Guadalupe González-Gallegos ${ }^{1}$ y Arturo Castro-Castro \\ Instituto de Botánica, Departamento de Botánica y Zoología, Centro Universitario de Ciencias Biológicas y \\ Agropecuarias, Universidad de Guadalajara, Zapopan, Jalisco, México. \\ ${ }^{1}$ Autor para la correspondencia: xanergo@hotmail.com
}

\begin{abstract}
Resumen: Se presenta el análisis florístico de un bosque de Abies y del bosque mesófilo de montaña adyacente, localizados al occidente de Jalisco, México. Se obtuvo una lista de plantas vasculares constituida por 80 familias, 195 géneros y 290 especies, con un índice de diversidad florística de 210.33 especies $/ \mathrm{km}^{2}$, el cual es elevado al compararlo con el obtenido en otras áreas boscosas similares. Las familias más diversas por cantidad de especies son Asteraceae (46), Fabaceae (22) y Orchidaceae (18). La flora de la comunidad estudiada es similar a lo descrito para los bosques de oyamel en Nueva Galicia y a los bosques mesófilos de la Faja Volcánica Transmexicana. Cinco especies recolectadas en el área se encuentran bajo alguna categoría de riesgo dentro de la Norma Oficial Mexicana NOM-059-SEMARNAT-2010 (Abies finckii, Comarostaphylis discolor subsp. discolor, Juglans major var. glabrata, Ostrya virginiana y Tilia americana var. mexicana). Los rodales de Abies flinckii son propuestos como área de conservación dado su carácter relictual.
\end{abstract}

Palabras clave: área natural protegida, Nueva Galicia, riqueza florística.

\begin{abstract}
A floristic analysis of a fir forest and the adjacent cloud forest located at western Jalisco was made. We obtained a list of vascular plants consisting of 80 families, 195 genera and 290 species, with a floristic diversity index of $210.33 \mathrm{species} / \mathrm{km}^{2}$, which is high when compared to other similar forested areas. The most diverse families in number of species are Asteraceae (46), Fabaceae (22) and Orchidaceae (18). This plant community is very similar to those described for fir forests in Nueva Galicia and the cloud forests from the Transmexican Volcanic Belt. There are five species in some risk category within the Norma Oficial Mexicana NOM-059-SEMARNAT-2010 (Abies flinckii, Comarostaphylis discolor subsp. discolor, Juglans major var. glabrata, Ostrya virginiana y Tilia americana var. mexicana). Patches of Abies flinckii are proposed as a conservation area due to its relictual condition.
\end{abstract}

Key words: floristic richness, natural protected area, Nueva Galicia.

E 1 género Abies Mill. (Pinaceae) se distribuye de forma insular en las zonas elevadas de las montañas del hemisferio norte y tiene cuatro centros de distribución geográfica: Mediterráneo, Siberia-Asia oriental, Norteamérica y México-Guatemala (Pérez de la Rosa, 1987; CisnerosLepe, 2005). En México, las especies de Abies ocupan nichos análogos a las especies boreales, pero también pueden tener adaptaciones o ecotipos de carácter subtropical y presentar una fitoasociación relativamente más diversa que estas (Guerrero-Hernández, 2012). Sin embargo, Manzanilla (1974) sugiere una riqueza baja. En México, los bosques de Abies u oyamel son considerados relictos de la extensa taiga (Rzedowski y McVaugh, 1966) que avanzó de norte a sur conforme los climas fríos descendieron hacia latitudes tropicales durante los periodos de glaciación.

En el territorio mexicano, estos bosques cubren entre 320 y 500 km² (Rzedowski, 1978; Cuevas-Guzmán et al., 2011), y las áreas más extensas y continuas se localizan en las serranías que circundan el Valle de México (Sánchez-González et al., 2006). Otra superficie considerable es la que cubren en el estado de Jalisco, donde se encuentran alrededor de 12,120 ha; es decir, entre el 24 y $38 \%$ de la cobertura nacional (PRODEFO, 1999). En la entidad, estos bosques ocupan tres áreas principales. Una de ellas se localiza al sureste del 
estado, en el Faja Volcánica Transmexicana (FVT), justo en los declives del Nevado de Colima, en los municipios de San Gabriel, Tuxpan y Zapotitlán de Vadillo, en donde se desarrollan Abies religiosa (Kunth) Schltdl \& Cham. y A. flinckii Rushfort (CONANP, 2006). Una segunda área, mucho más extensa, se encuentra en el occidente del estado en la Sierra Madre del Sur (SMS), desde las sierras de El Cuale, Jolapa y San Sebastián del Oeste, en los municipios de Talpa de Allende, Mascota y San Sebastián del Oeste, respectivamente; hasta la Sierra de Cacoma, en los municipios de Atenguillo y Cuautla; y la Sierra de Manantlán, en los municipios de Autlán de Navarro y Cuautitlán de García Barragán; es decir, desde las partes altas de la cuenca del Río Ameca hasta las mayores elevaciones de las cuencas de los ríos Ayuquila, Armería y Marabasco. Esta región representa, en su mayor parte, una faja de pinares y encinares húmedos con manchones, a veces muy espaciados, de bosque de oyamel (Rzedowski y McVaugh, 1966), donde A. flinckii y A. religiosa presentan una distribución geográfica importante pero restringida. Además, estas especies de oyamel forman un archipiélago en la vertiente pacífica de la SMS y de la FVT (J.A. Pérez de la Rosa, com. pers.), y se consideran elementos relictuales. En contraste, Vázquez et al. (2000) resaltan la importancia de bosques puros de oyamel encontrados en las cercanías de la laguna de Juanacatlán, Mascota. Otra área de distribución de Abies en Jalisco se localiza en la porción sur de la Sierra Madre Occidental (SMO), al norte del estado, en el municipio de Mezquitic. La región corresponde a la Sierra de Bolaños, donde se desarrolla $A$. durangensis Martínez asociado a bosques de Pinus-Quercus-Alnus (Pérez de la Rosa, 1987; McVaugh, 1992; Luquín et al., 2004).

La flora y vegetación de la porción occidental de Jalisco es poco conocida (Rzedowski y McVaugh, 1966; Reynoso-Dueñas et al., 2006). En esta área confluyen los reinos biogeográficos Neártico y Neotropical (Holt et al., 2013), y es parte de la zona que Morrone (2005) denominó como Zona de Transición Mexicana. Además, es un área de traslape entre la FVT y la SMS. Como resultado, la diversidad litológica, geológica, morfotectónica, paisajística y biológica de la zona, es elevada (Vázquez et al., 2000; Reynoso-Dueñas et al., 2006). La complejidad de la región se refleja en una diversidad florística alta para numerosas formaciones vegetales, entre ellas, los bosques de Abies (Cuevas-Guzmán et al., 2011).

Aunque los bosques de oyamel neotropicales han despertado gran interés científico por su diversidad florística, distribución relictual en las altas montañas cerca del límite de la vegetación arbórea (Cuevas-Guzmán et al., 2011) y por su contacto frecuente con los bosques mesófilos de montaña (BMM), en Jalisco han sido poco estudiados (Cuevas-Guzmán et al., 2011 ofrecen una revisión amplia al respecto). En su mayoría, los estudios acerca de estas comunidades en la entidad han sido enfocados al análisis estructural (RamosHerrera, 1991; Figueroa y Moreno, 1993; Cisneros-Lepe,
2005; Cuevas-Guzmán et al., 2011), mientras que el aspecto florístico se ha dirigido al estrato arbóreo (Cisneros-Lepe, 2005). En otros casos, forman parte de investigaciones más amplias, en las que el oyamel no es el objeto de estudio (Vázquez-García et al., 2000). Aún más, trabajos con enfoques biogeográficos en bosques de oyamel mexicanos son escasos a nivel de género (Madrigal-Sánchez, 1967; Rzedowski, 1978; Jaramillo-Correa et al., 2008) y no existen en el nivel de especie, excepto el trabajo de Sánchez-González et al. (2006). En conclusión, en Jalisco no existen trabajos orientados al análisis florístico de estas comunidades.

Por otro lado, los análisis e inventarios florísticos son herramientas básicas en el establecimiento y ejecución de programas adecuados de aprovechamiento y conservación (Humphries et al., 1991). Es por ello que el presente estudio tuvo como objetivo analizar la composición florística, además de describir de forma cualitativa y fisonómica el bosque de Abies y el BMM adyacente, en el cual se extiende la presencia del oyamel, en Juanacatlán, Jalisco.

\section{Material y métodos}

Área de estudio. El área de estudio se encuentra en el occidente del estado de Jalisco y corresponde a una fracción de la parte alta de la microcuenca El Copal, en las cercanías de la laguna de Juanacatlán, Mascota. Se localiza entre las coordenadas $20^{\circ} 36^{\prime} 26^{\prime \prime}$ - $20^{\circ} 38^{\prime} 07^{\prime \prime} \mathrm{N}$ y $104^{\circ} 41^{\prime} 34^{\prime}$ ' - $104^{\circ}$ 42' 47' O, en la región administrativa Sierra Occidental y al este de la región Costa Norte, $13 \mathrm{~km}$ al noreste de Mascota. Su acceso es por la brecha Mascota-Navidad-Juanacatlán. Tiene una superficie de 397 ha y se registra una cota altitudinal que va de los 2,100 a los 2,400 m, con numerosas cañadas y laderas. Cerca de 90 ha se encuentran dentro del Área de Protección de Recursos Naturales de la Cuenca Alimentadora del Distrito Nacional de Riego 043, fracción Sierra de Vallejo-Río Ameca (CONANP, 2014), y se ubica dentro de la Región Hidrológica Río Ameca y en la Cuenca Hidrológica Ameca-Ixtapa (CEA, 2012; Figura 1).

El área está situada dentro de la FVT (Ferrusquía-Villafranca, 1998), en una zona donde converge con algunos afloramientos ígneos intrusivos de la SMS (Gómez-Tuena et al., 2005). También forma parte de un espacio limítrofe de las subprovincias fisiográficas Sierras de la costa de Jalisco y Colima, y Sierras de Jalisco (SPP, 1981). De acuerdo a las divisiones florísticas de México, propuestas por Rzedowski (1978), la zona es ubicada dentro de la provincia de las Serranías Meridionales y estas a su vez, en la región Mesoamericana de Montaña. Según el criterio biogeográfico de Morrone (2005), el área se localiza entre los límites del Eje Volcánico Transmexicano y la Costa Pacífica Mexicana.

El origen de esta serranía es producto de vulcanismo máfico intermedio, que se extendió del Plioceno tardío al Cuaternario (Gómez-Tuena et al., 2005). El material parental se constituye de rocas ígneas extrusivas ácidas, así como 
de suelos aluviales en una parte de la microcuenca y suelo residual en la localidad del Ixtololo (CETENAL, 1974). Los suelos predominantes son del tipo andosol, cambisol y regosol (CETENAL, 1975). El clima de acuerdo a Köppen modificado por García (2004) es templado subhúmedo con verano fresco largo y con lluvias en verano con un porcentaje de lluvia invernal $<5 \%$, el más húmedo de los subhúmedos [C $\left(\mathrm{w}_{2}\right)(\mathrm{w})$; SPP, 1980]. El mes más cálido tiene una temperatura media de $20^{\circ} \mathrm{C}$, que se presenta antes del solsticio de verano y, la temperatura media del mes más frío es de $6^{\circ} \mathrm{C}$. Tiene una temperatura media anual de 13.8 a $14.6{ }^{\circ} \mathrm{C}$ y precipitación media anual de 1,200 mm (Hijmans et al. 2005).

Trabajo de campo. Desde octubre del 2010 a septiembre de 2011 se realizaron doce excursiones a la zona de estudio, una cada mes y con una duración de tres días cada una. En cada salida se realizaron recolectas intensivas de la flora vascular, de esta manera se trató de abarcar el área completa del polígono. Los ejemplares recolectados se procesaron mediante técnicas convencionales para cada grupo taxonómico (Lot y Chiang, 1986). El juego principal de la colección fue depositado en el herbario IBUG, duplicados se distribuyeron a los herbarios IEB, MEXU y ZEA (acrónimos de acuerdo a Thiers, 2011).

Trabajo de gabinete. Para la identificación del material colectado y su distribución geográfica se utilizaron claves y descripciones taxonómicas disponibles en floras regionales, monografías y revisiones taxonómicas (McVaugh, 1961, 1984, 1985, 1987, 1989, 1992; González-Villarreal, 1986, 1990, 1996a, b, 2000a, b, c, 2001, 2002; Calderón de Rzedowski, 1991, 1992; Graham, 1994; Rzedowski y Calderón de Rzedowski, 1995, 2004; Fernández, 1996; Villarreal, 2000; Calderón de Rzedowski y Rzedowski, 2001: Patiño, 2001; Ocampo, 2003; Mickel y Smith, 2004; Pérez-Calix y Carranza, 2005; Carranza, 2007, 2008; Castillejos-Cruz y Solano, 2008; Pérez-Calix, 2009; Villarreal-Q. y EstradaC., 2011). La afinidad fitogeográfica de los géneros se cotejó con base en la distribución señalada para cada uno de ellos en la base de datos Tropicos (2011) y de acuerdo a las categorías utilizadas por Gentry (1982) y, Quintana-Ascencio y Gonzalez-Espinosa (1993).

Las familias, géneros y especies se arreglaron en orden alfabético de acuerdo a las propuestas de APG III (2009) para angiospermas, Christenhusz et al. (2011a) para gimnospermas y Christenhusz et al. (2011b) para helechos y afines. Las angiospermas fueron organizadas, en primer término, en magnolídeas, monocotiledóneas y eudicotiledóneas, de acuerdo a los principales clados avalados por APG III (2009). Fueron consultadas las bases de datos Tropicos (2011) e IPNI (2013) para corroborar la asignación de géneros por familias y la ortografía correcta de los nombres científicos. El estado de riesgo de las especies se determinó con base en la lista de la Norma Oficial Mexicana NOM-
059-SEMARNAT-2010 (SEMARNAT, 2010). Las formas biológicas por especie se asignaron con base en las definiciones de FontQuer (1953) y Moreno (1984), de acuerdo a una clasificación provisional utilizada por Frías-Castro et al. (2013), en la que se designan categorías con base en tres atributos: (1) hábito: árbol, arbusto, bejuco o liana, hierba; (2) hábitat: epífito, rupícola, terrestre; y (3) tipo de nutrición: autótrofa, hemiparásita, holoparásita y saprófita. La descripción de la vegetación se realizó de forma cualitativa sustentada en las observaciones realizadas en campo para las especies dominantes, su distribución y su fisonomía. La clasificación sigue las propuestas de Rzedowski y McVaugh (1966) y, Rzedowski (1978).

Por último, para comparar la diversidad taxónomica $(B)$ entre áreas de diferentes tamaños se siguió la propuesta de Squeo et al. (1998), en donde se analiza el número de especies registradas dividido por el logaritmo natural del área en $\mathrm{km}^{2}: B=S / \ln A$, donde $S$ es el número de especies registradas y $A$ es el área.

\section{Resultados}

Composición florística. La flora vascular asociada a la población de Abies flinckii comprende 80 familias, 195 géneros y 290 especies (Apéndice 1, Cuadro 1). Está representada por helechos y afines, gimnospermas y angiospermas. El primer grupo incluye miembros de cuatro de las cinco subclases propuestas por Christenhusz et al. (2011a) y suma 20 especies (Cuadro 1). Las gimnospermas manifiestan una menor riqueza, mientras las angiospermas concentran el mayor número de especies (Apéndice 1, Cuadro 1). Las eudicotiledóneas contienen $78.8 \%$ de la riqueza de angiospermas, las monocotiledóneas el 29.4\%; las magnolídeas representan el 7.6\%. Las familias mejor representadas fueron: Asteraceae con 46 especies (16.1\%), Fabaceae 22 (7.6\%), Orchidaceae 18 (6.2\%), Lamiaceae 14 (4.8\%), Solanaceae 11 (3.8\%), Polypodiaceae y Poaceae 8 ( $2.7 \%$, cada una; Cuadro 2$)$, que en conjunto representan $44 \%$ de la riqueza total. Los géneros más diversos son Ageratina, Cuphea, Desmodium, Polypodium, Quercus, Salvia y Solanum (Cuadro 2).

Cuadro 1. Riqueza de la flora vascular de un bosque de Abies y el bosque mesófilo de montaña adyacente en Juanacatlán, Mascota, Jalisco.

\begin{tabular}{lllll}
\hline Grupo & Clases & Familia & Géneros & Especies \\
\hline Helechos y afines & 4 & 8 & 12 & 20 \\
Gimnospermas & 1 & 1 & 2 & 6 \\
Angiospermas & & & & \\
Magnolídeas & 1 & 1 & 2 \\
Monocotiledóneas & 11 & 41 & 54 \\
Eudicotiledóneas & 59 & 139 & 208 \\
Total & $\mathbf{8 0}$ & $\mathbf{1 9 5}$ & $\mathbf{2 9 0}$ \\
\hline
\end{tabular}


Cuadro 2. Familias y géneros más diversos de un bosque de Abies y el bosque mesófilo de montaña adyacente en Juanacatlán, Mascota, Jalisco.

\begin{tabular}{llll}
\hline Familias & Especies & Géneros & Especies \\
\hline Asteraceae & 46 & Salvia & 9 \\
Fabaceae & 22 & Cuphea & 6 \\
Orchidaceae & 18 & Quercus & 6 \\
Lamiaceae & 14 & Solanum & 6 \\
Solanaceae & 11 & Ageratina & 5 \\
Polypodiaceae & 8 & Desmodium & 5 \\
Poaceae & 8 & Polypodium & 5 \\
\hline
\end{tabular}

La forma biológica por hábito más común fue la de hierbas, con un total de 200 especies (62.8\%), luego los árboles con $43(14.6 \%)$, arbustos $41(13.9 \%)$ y bejucos $11(3.72 \%)$. Con base en el hábitat, las plantas rupícolas representan $3.72 \%$ (11 especies), las epífitas el $6.78 \%$ (20), y el resto corresponde a las terrestres. En cuanto a tipo de nutrición, se registraron dos especies hemiparásitas y una saprófita, el resto son autótrofas (Cuadro 3). El área de estudio presenta una diversidad taxonómica de 210.3 especies $/ \mathrm{km}^{2}$.

Tipos de vegetación. En el área se presentan dos tipos de vegetación, bosque de Abies y bosque mesófilo de montaña (Figura 1). Es importante notar que los límites para diferenciar una comunidad de otra no son abruptos, sino que su transición es gradual. Además hay una faja pequeña de pinares y encinares húmedos que se mezclan en pocos espacios con el BMM.

Bosque de Abies.- Se presenta en la parte alta de las cañadas y forma un rodal de casi 50 ha. Su fisiografía presenta poca pendiente y se encuentra entre 2,340 y 2,420 m de elevación (Figuras 1,2). Aquí prevalece una humedad ambiental alta, por lo que es común observar niebla hasta en los meses más secos y fríos. Es claro que esta comunidad tiende a ser más homogénea conforme aumenta la elevación, y la especie que domina es Abies flinckii. El estrato arbóreo superior va de los 20 a $40 \mathrm{~m}$. En el estrato medio los árboles tienen

Cuadro 3. Diversidad de hábito, tipo de hábitat y nutrición de las plantas de un bosque de Abies y el bosque mesófilo de montaña adyacente en Juanacatlán, Mascota, Jalisco. Existe más de un hábito para algunas especies, por lo que se cuentan en más de una ocasión y no coincidirá con las 290 especies registradas.

\begin{tabular}{ll|ll|ll}
\hline Hábito & Especies & $\begin{array}{l}\text { Tipo de } \\
\text { hábitat }\end{array}$ & Especies & $\begin{array}{l}\text { Tipo de } \\
\text { nutrición }\end{array}$ & Especies \\
\hline Árbol & 43 & Terrestre & 177 & Autótrofo & 287 \\
Arbusto & 41 & Epífito & 18 & Hemiparásito & 2 \\
Hierba & 200 & Rupícola & 5 & Saprófito & 1 \\
Trepadora & 11 & & & & \\
\hline
\end{tabular}

alturas de 10-20 m y continúa dominante Abies finckii en asociación con Arbutus xalapensis, Alnus jorullensis subsp. jorullensis, Clethra hartwegii, Pinus montezumae, Prunus serotina subsp. capuli, Quercus castanea, Q. obtusata y escasos árboles de Symplocos citrea. Un estrato arbóreo inferior que oscila entre 4 y 10 m está dominado por Styrax ramirezii, y con menor frecuencia aparecen Arbutus tessellata, Cornus disciflora, Garrya longifolia, Symplocos citrea y en algunos sitios un poco más abiertos es común Podachaenium eminens (Figura 2).

El estrato arbustivo en algunos espacios es denso y entre las especies más comunes destacan Arctostaphylos pungens, Baccharis heterophylla, Brickellia squarrosa var. oligadena, Buddleja sessiliflora, Cestrum terminale, Cosmos carvifolius, Fuchsia obconica, Lonicera pilosa y Solanum brachystachys. En los márgenes del bosque donde se presentan claros y vegetación secundaria se puede encontrar a Triumfetta columnaris. El estrato herbáceo está bien representado por Asclepias pringlei, Bidens chiapensis var. feddemana, Cirsium ehrenbergii, Drymaria multiflora, Euphorbia sphaerorhiza, Geranium lilacinum, Gnaphalium americanum, Macromeria pringlei, Oxalis hernandezii, Physalis orizabae, Plantago australis, Psacalium eriocarpum, Ranunculus mexiae, Salvia elegans, S. laevis, Sprekelia formosissima, Tigridia multiflora, Valeriana urticifolia y Viola grahamii. Aquí, las apiáceas se encuentran bien representadas por Donnellsmithia juncea, Eryngium mexiae, Neogoezia planipetala y una especie de Prionosciadium aún no determinada. Entre las orquídeas es evidente la presencia de Govenia liliacea, la saprófita Hexalectris grandiflora, y Oestlundorchis eriophora. Los helechos por su parte se encuentran representados por Adiantum andicola, Asplenium cuspidatum, A. monanthes y Pleopeltis polylepis, este último es epífito, incluso en los oyameles.

Bosque mesófilo de montaña.- Es la vegetación dominante en el área delimitada (306 ha), también cubre la mayor superficie de esta fracción de la microcuenca (Figura 1). El BMM presenta diversas asociaciones, que a menudo difieren entre sí en cuanto a la altura, la fenología y sobre todo, en las especies dominantes. En la zona de estudio, se encuentra en áreas considerablemente grandes a lo largo de las laderas y cañadas e incluso en la parte alta de las mismas, donde el terreno tiende a ser más plano y con menos pendiente. Sus límites altitudinales van de 2,100 a 2,360 m (Figura 2).

El estrato emergente presenta árboles que tienen alturas mayores de $30 \mathrm{~m}$. Las especies dominantes son Abies flinckii (algunos miden hasta 50 m o más), Quercus obtusata, $Q$. scytophylla, Pinus devoniana y P. lumholtzii. En el estrato arbóreo superior, que va de los 20 a $30 \mathrm{~m}$, se encuentran las especies mencionadas anteriormente, además de Clethra hartwegii, Meliosma dentata, Quercus candicans, Tilia americana var. mexicana, y en ocasiones Cornus disciflora, 


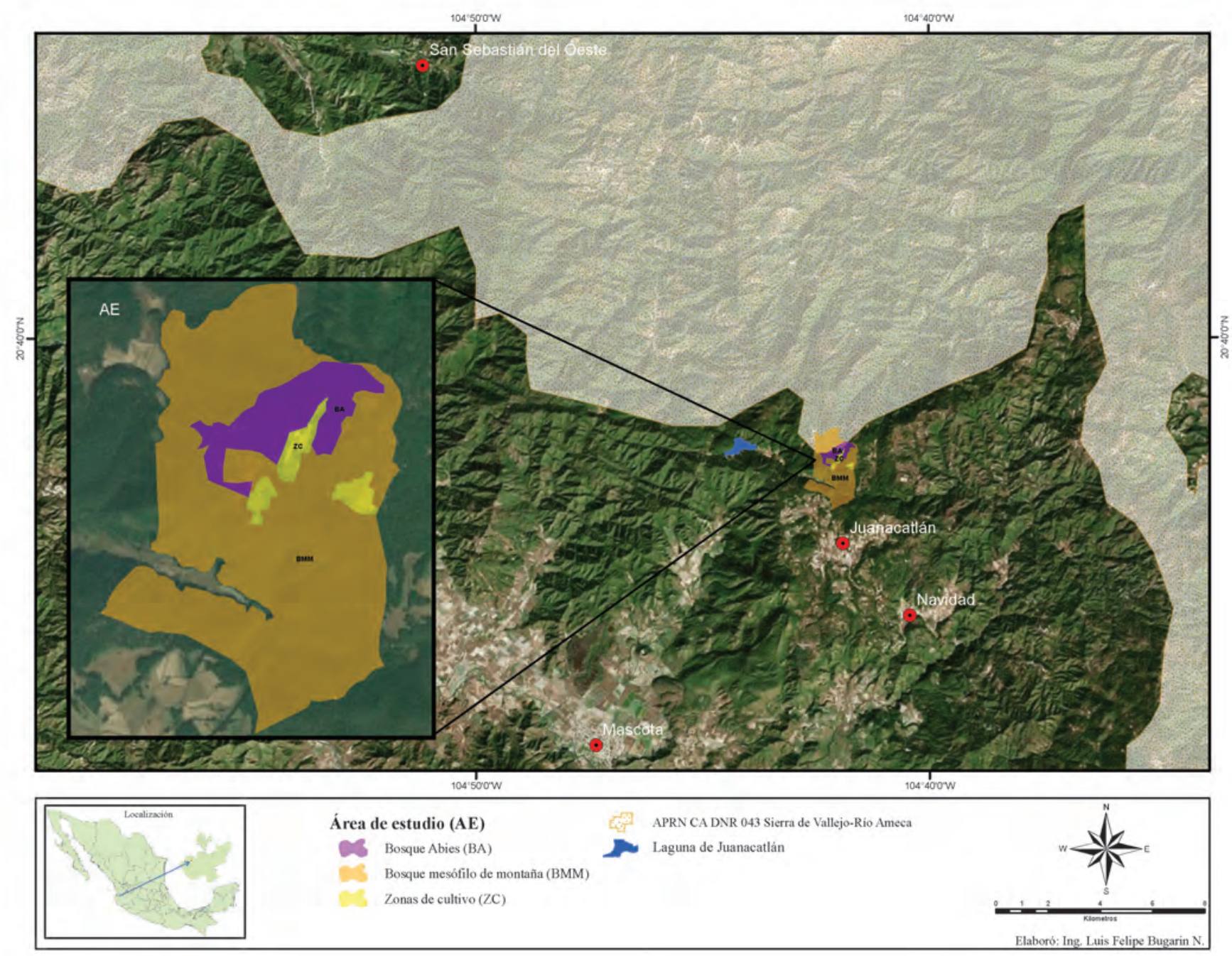

Figura 1. Mapa de localización de un bosque de Abies y el bosque mesófilo de montaña adyacente en Juanacatlán, Mascota, Jalisco, y tipos de vegetación identificados (elaborado por Luis F. Bugarín-Navarro, 2013).

Oreopanax xalapensis y Symplocos citrea alcanzan estas magnitudes. En el siguiente estrato, se distinguen árboles de 10 a 20 m de altura, representados en su mayoría por Styrax ramirezii y Symplocos citrea y, con menos frecuencia, Arbutus xalapensis, Cleyera integrifolia, Cornus disciflora, Myrsine juergensenii y Prunus serotina subsp. capuli. Sólo en un par de cañadas se encontró una población de Ostrya virginiana, cuyos individuos alcanzan hasta $20 \mathrm{~m}$ de altura. Por el borde de algunos arroyos estacionales se desarrollan Ilex tolucana y Salix jaliscana, además de individuos aislados de Juglans major. En el estrato arbóreo inferior los árboles van de 4 a 10 m de altura, entre ellos figuran Dendropanax arboreus, Garrya longifolia, Rhamnus hintonii, Telanthophora jaliscana y Ternstroemia lineata subsp. lineata (Figura 2).

El estrato arbustivo está compuesto por Cestrum terminale, Fuchsia obconica, Monnina xalapensis, Rumfordia floribunda, Salvia gesneriiflora, Solanum brachystachys y Viguiera ensifolia. En el estrato herbáceo destacan Astra- galus jaliscensis, Begonia gracilis, Bidens chiapensis var. feddemana, Cosmos bipinnatus, C. sessilis, Cuphea jorullensis, C. hookeriana, Donnellsmithia mexicana, Echeandia coalcomanensis, Gentianopsis detonsa var. lanceolata, Geranium lilacinum, Oxalis hernandezii, Polygala compacta, Psacalium eriocarpum, Salvia elegans, S. thyrsiflora y Stellaria cuspidata. Las epífitas están representadas por las orquídeas Epidendrum neogaliciense, Homalopetalum pachyphyllum, Laelia autumnalis, Pleurothallis sanguinolenta y Stelis villosa, así como las bromeliáceas Tillandsia bourgaei y $T$. calothyrzus, esta última es común en las ramas y troncos de los oyameles. Los helechos Asplenium monanthes, Phlebodium areolatum, Pleopeltis angusta, $P$. polylepis y Polypodium madrense, así como la piperácea Peperomia galioides (con distribución muy localizada en el área) forman parte de este hábito. Las orquídeas terrestres Bletia purpurata, Govenia superba y Malaxis brachyrhynchos también crecen en esta comunidad vegetal. Entre las rupícolas se pue- 


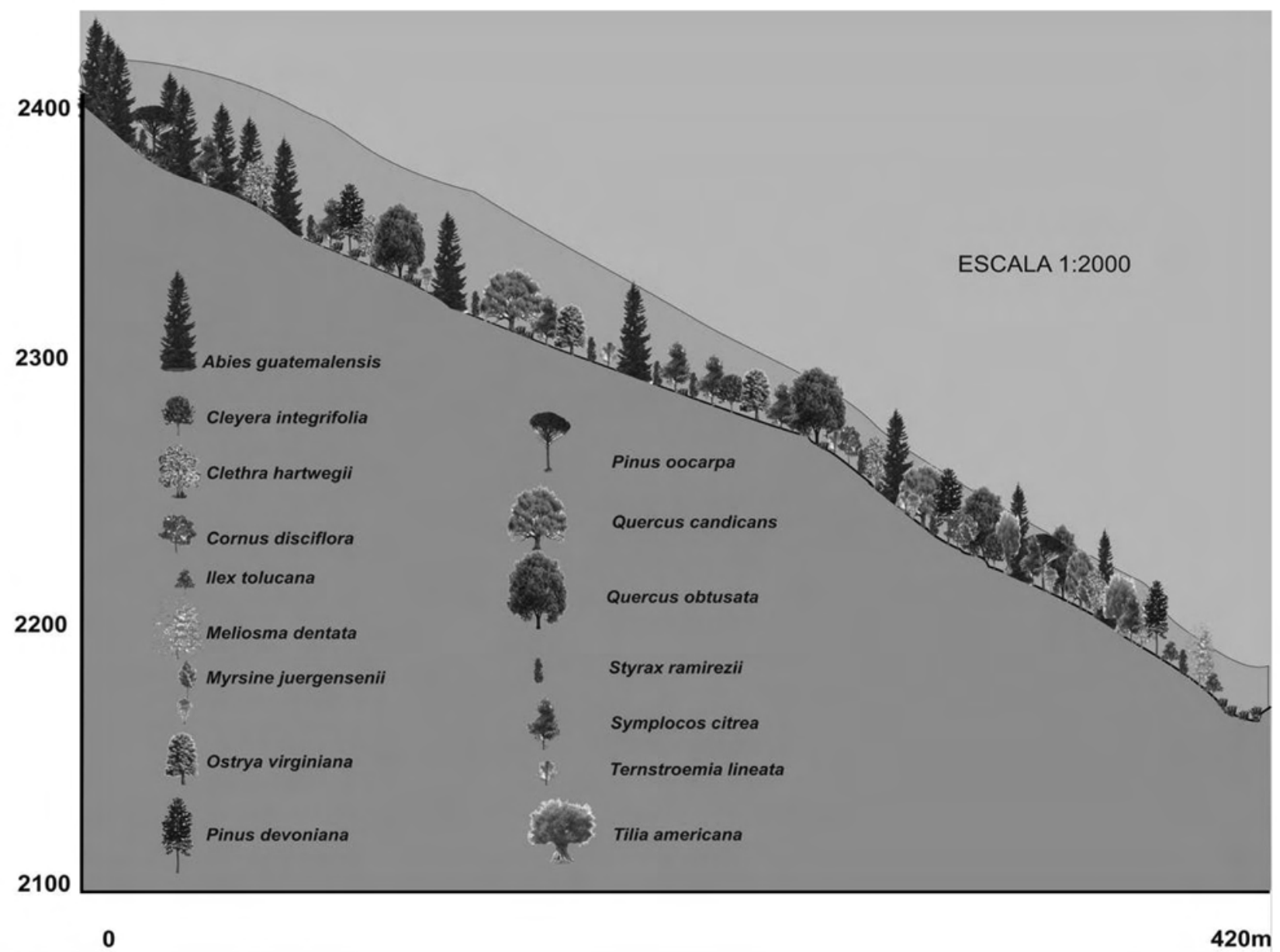

Figura 2. Perfil esquemático de la vegetación de una cañada del bosque de Abies y el bosque mesófilo de montaña adyacente en Juanacatlán, Mascota, Jalisco.

de nombrar a Oncidium graminifolium y Asplenium cuspidatum. Las trepadoras o bejucos están representadas por $\mathrm{Me}$ tastelma latifolium, Passiflora exsudans, Smilax moranensis, Solanum appendiculatum y Toxicodendron radicans.

Dentro del bosque hay áreas con vegetación secundaria, cultivos y aprovechamientos forestales, que han ocasionado un cambio en la composición florística y fisonomía natural del bosque. En los sitios con aprovechamiento forestal se encuentra a Alnus jorullensis subsp. jorullensis, Crataegus pubescens, Lippia umbellata, Quercus castanea, Q. obtusata, Symplocos citrea y también algunos árboles de Abies flinckii. El estrato arbustivo es denso y destacan Baccharis heterophylla, Cestrum terminale, Heterotheca inuloides, Salvia mexicana, Solanum lanceolatum y Triumfetta columnaris. Las hierbas son abundantes y las especies más frecuentes son Bidens chiapensis var. feddemana, Melampodium perfoliatum, Penstemon miniatus subsp. apateticus, Solanum nigrescens, S. stoloniferum, Valeriana urticifolia subsp. urticifolia y Verbena carolina.

En los espacios con cultivos donde casi toda la cubierta vegetal ha sido removida, se han establecido especies que podrían considerarse pioneras. Aquí destacan Acalypha multispicata, Allium kunthii, Argemone ochroleuca subsp. ochroleuca, Avena sativa, Crotalaria sagittalis, Echeandia imbricata, Heterotheca inuloides, Kyllinga pumila, Mecardonia procumbens, Oenothera pubescens, Oxalis corniculata, Phacelia platycarpa, Pinaropappus roseus, Salvia longispicata, Stachys agraria, Solanum nigrescens y S. stoloniferum. Son frecuentes los pastos Panicum bulbosum, Piptochaetium virescens y la orquídea Dichromanthus aurantiacus. Por último, en sitios con suelos mal drenados se identificó una población grande de Equisetum hyemale var. affine.

Afinidades fitogeográficas de la flora arbórea. Un atributo notable de los bosques mesófilos de montaña y de Abies, es que los diferentes estratos incluyen elementos de afinidades fitogeográficas distintas. La composición arbórea en el área de estudio permite reconocer que el 55\% de las especies tienen afinidad holártica. Por su parte, $21 \%$ de los elementos tienen afinidad con el este de Asia, pero algunas especies también se desarrollan en el este de Norteamérica (por ejemplo Ostrya virginiana y Tilia americana var. mexicana), y otros géneros y especies sudamericanos de afinidad 
neotropical andina (p. ej. Cleyera, Ternstroemia y Juglans major var. glabrata). Los elementos neotropicales andinos constituyen el 24\% de las especies, y se incluyen los taxa de afinidad con el este de Asia. Por último, el 7\% es de afinidad pantropical, así como de África.

Endemismo de la flora. El área de estudio está inmersa dentro del centro de endemismo Talpa-Cuale (Atenguillo, Mascota, Talpa de Allende y San Sebastián del Oeste), identificado por Hernández-López (1995) en el estado de Jalisco. Para el bosque de Juanacatlán, el 35.05\% (102) de las especies son endémicas a México, $4.81 \%$ (14) son exclusivas de Jalisco y $3.78 \%$ (11) representan endemismos para las serranías del occidente del estado (Apéndice 1).

Por otro lado, destacan como microendemismos dos especies nuevas descubiertas con anterioridad en regiones aledañas a la zona de estudio. Una de ellas, Cunila jaliscana (Lamiaceae), ha sido recientemente publicada (García-Peña y González-Gallegos, 2013), mientras que una segunda perteneciente al género Prochnyanthes (Agavaceae) se encuen- tra en proceso de publicación. También, fueron identificadas en el área de estudio la rubiácea Chiococca phaenostemon y la asparagácea Echeandia coalcomanensis, que exhiben una distribución restringida y representan registros nuevos para la flora de Jalisco (Apéndice 1).

Estado de riesgo de las especies. De acuerdo con la Norma Oficial Mexicana NOM-059-SEMARNAT-2010 (SEMARNAT, 2010), una de las especies encontrada en el área se encuentra en riesgo de extinción, Tilia americana var. mexica$n a$. Por su parte, bajo protección especial se señalan a Abies flinckii y Comarostaphylis discolor subsp. discolor. En la categoría de amenazadas están Juglans major var. glabrata y Ostrya virginiana (Apéndice 1).

\section{Discusión}

El conocimiento florístico del bosque de la localidad de Juanacatlán era muy escueto o casi nulo. Al igual que en otras sierras del occidente de Jalisco, en Juanacatlán las explora-

Cuadro 4. Comparación de la riqueza florística entre el bosque de Abies y el bosque mesófilo de montaña adyacente en Juanacatlán, Mascota, Jalisco, y otros bosques o poblaciones de Abies en México.

\begin{tabular}{|c|c|c|c|c|}
\hline & Localidad & Fuente & Gradiente altitudinal $(\mathrm{m})$ & Especies \\
\hline 1 & $\begin{array}{l}\text { Reserva de la biósfera Mariposa Monarca, Estado de México } \\
\text { y Michoacán }\end{array}$ & Cornejo-Tenorio et al., 2003 & $2,400-3,300$ & 225 \\
\hline 2 & Sierra de Monte Alto, Estado de México & Osorio-Rosales, 1984 & $2,950-3,500$ & 172 \\
\hline 3 & Sierra del Ajusco, Distrito Federal & $\begin{array}{l}\text { Álvarez del Castillo, 1987; } \\
\text { Nieto, } 1995\end{array}$ & $2,970-3,500$ & 66 \\
\hline 4 & Sierra Chichinautzin, Distrito Federal & Velázquez y Cleef, 1993 & $3,000-3,550$ & 78 \\
\hline 5 & $\begin{array}{l}\text { Parque Nacional Izta-Popo, Estado de México, } \\
\text { Puebla y Morelos }\end{array}$ & $\begin{array}{l}\text { May-Nah, 1971; Boyas, 1993; } \\
\text { Almeida-Leñero, } 1997\end{array}$ & $2,800-3,600$ & 104 \\
\hline 6 & Parque Nacional Zoquiapan, Estado de México y Puebla & Vega-Aviña, 1982 & $3,150-3,500$ & 70 \\
\hline 7 & Cerro Tláloc, Estado de México & Sánchez-González, 2004 & $3,100-3,500$ & 138 \\
\hline 8 & Sierra de Calpulalpan, Tlaxcala & $\begin{array}{l}\text { Madrigal-Sánchez, 1967; } \\
\text { Castillejos-Cruz y } \\
\text { Ramírez-Ramírez, } 1992\end{array}$ & $2,800-3,810$ & 75 \\
\hline 9 & Sierra de Tlaxco-Tequesquitla, Tlaxcala & $\begin{array}{l}\text { Madrigal-Sánchez, 1967; } \\
\text { Castillejos-Cruz y } \\
\text { Ramírez-Ramírez } 1992\end{array}$ & $2,800-3,810$ & 114 \\
\hline 10 & Parque Nacional La Malinche, Tlaxcala & $\begin{array}{l}\text { Madrigal-Sánchez, 1967; } \\
\text { Castillejos-Cruz y } \\
\text { Ramírez-Ramírez, } 1992\end{array}$ & $2,800-3,810$ & 113 \\
\hline 11 & Parque Nacional El Chico, Hidalgo & $\begin{array}{l}\text { Madrigal-Sánchez, 1967; } \\
\text { Hernández-Rosales, } 1995\end{array}$ & $2600-3086$ & 77 \\
\hline 12 & Parque Nacional Cofre de Perote, Veracruz & Narave, 1985 & $3,000-3,500$ & 98 \\
\hline 13 & Arroyo El Infierno, Pueblo Nuevo, Durango & García-Arévalo, 2008 & $2,500-2,750$ & 199 \\
\hline 14 & Sierra de Zapalinamé, Coahuila & Encina-Domínguez et al., 2008 & $2,668-3,025$ & 73 \\
\hline 15 & Sierra de San Carlos, Tamaulipas & Briones, 1991 & $1,300-1,400$ & 60 \\
\hline 16 & Juanacatlán, Mascota, Jalisco & Este trabajo & $2,100-2,400$ & 290 \\
\hline
\end{tabular}


RicARDo GuerRero-HernándeZ ET AL.

Cuadro 5. Comparación de la riqueza florística de un bosque de Abies y el bosque mesófilo de montaña adyacente en Juanacatlán, Mascota, Jalisco, con otras de vegetación similar, utilizando la diversidad taxonómica ( $B$; modificado a partir del Cuadro 1 en López-Pérez et al., 2011). aEstos datos de superficie fueron tomados de López-Pérez et al. (2011), no están señalados en los trabajos originales de Luna et al. (1988, 1989). bLa superficie del bosque mesófilo fue tomada de Meave et al. (1992), donde se estima que del área total de Omiltemi (3,600 ha), el 20\% corresponde a esta vegetación.

\begin{tabular}{|c|c|c|c|c|c|}
\hline Localidad & Elevación (m) & Área $\left(\mathrm{km}^{2}\right)$ & $\begin{array}{l}\text { Número de } \\
\text { especies }\end{array}$ & $B(\mathrm{sp} / \ln A)$ & Fuente \\
\hline Teocelo, Veracruz & $1,100-1,630$ & $21.00^{\mathrm{a}}$ & 277 & 90.98 & Luna et al., 1988 \\
\hline Ocuilán, Morelos y Estado de México & $1,800-2,400$ & $12.20^{\mathrm{a}}$ & 160 & 63.96 & Luna et al., 1989 \\
\hline Omiltemi, Guerrero & $2,300-2,700$ & $7.20^{b}$ & 244 & 123.60 & Jiménez et al., 1993 \\
\hline Molote, Guerrero & $2,300-2,500$ & 7.00 & 458 & 235.37 & Lozada et al., 2003 \\
\hline Lolotla, Hidalgo & $1,050-1,580$ & 10.22 & 359 & 154.45 & Ponce-Vargas et al., 2006 \\
\hline Minatitlán, Colima & $1,400-1,860$ & 1.94 & 247 & 372.72 & Padilla et al., 2008 \\
\hline Valle de Bravo, Estado de México & $2,000-2,620$ & 18.70 & 391 & 133.51 & López-Pérez et al., 2011 \\
\hline Juanacatlán, Mascota, Jalisco & $2,100-2,400$ & 3.97 & 290 & 210.33 & Este trabajo \\
\hline
\end{tabular}

ciones botánicas habian sido esporádicas y por lo general dirigidas hacia un grupo biológico particular. El presente trabajo representa el primer esfuerzo exhaustivo por obtener un inventario florístico del área.

La riqueza florística encontrada representa entre 4 y $10 \%$ del total de especies estimado para el bosque mesófilo de montaña en México por Villaseñor (2010) y Rzedowski (1991), respectivamente. Con base en los resultados de Villaseñor (2010), el área de estudio resguarda 10\% de la flora del bosque mesófilo de montaña encontrada en Jalisco. Asimismo, se encuentran 35.2\% de las familias, $6.1 \%$ de los géneros y $4.3 \%$ de las especies registradas para el estado de Jalisco (Ramírez-Delgadillo et al., 2010). Las seis familias más diversas de angiospermas encontradas en el área de estudio están dentro del listado de 15 familias con mayor riqueza en México (Villaseñor, 2003). Las familias Cactaceae, Euphorbiaceae y Rubiaceae, las cuales son de considerable riqueza en el país, están poco representadas en el área. Por otra parte, Lamiaceae muestra mayor riqueza que Poaceae. La proporción de la riqueza por familia es similar a la observada en otros bosques mesófilos de montaña (Rzedowski, 1996; Alcántara y Luna, 2001, Lozada et al., 2003).

La flora del área de estudio concentra en 13 familias el $57 \%$ de las especies, resultado que dista del $72 \%$ que es contenido por 14 familias en el bosque de oyamel en el Cerro Tláloc (Sánchez-González et al., 2006), que de manera indirecta refleja una mayor equitatividad. No obstante, se ajusta mejor al de un bosque mesófilo como el de Lolotla, Hidalgo (Ponce-Vargas et al., 2006), en el cual 48\% de las especies son representadas por 13 familias.

De los siete géneros más diversos en el área, solamente Cuphea y Polypodium no están dentro de los 20 géneros de plantas vasculares con mayor cantidad de especies de México (Villaseñor, 2004). Aunque, Polypodium es registrado como uno de los principales géneros de plantas epífitas en el BMM (Rzedowski, 1996).
El porcentaje de especies por tipo de hábito coincide con el patrón general observado para los géneros de plantas vasculares de México (Villaseñor, 2004). En comparación con otros BMM, la proporción de plantas epífitas (20 especies, 6.89\%) registrada en el área es una de las más bajas (Meave et al., 1992; García, 1993; Rzedowski, 1996; Alcántara y Luna, 1997, 2001; López-Pérez et al., 2011; Cuadro 4). Sin embargo, es más alta que los bosques más puros de Abies de la FVT y la SMO (Cornejo-Tenorio et al., 2003; SánchezGonzález et al., 2006; Encina-Domínguez et al., 2008), y a lo reportado por Alcántara y Luna (2001), para cada una de las dos localidades de BMM que estudiaron (17 y 15 respectivamente). Lo anterior difiere a lo descrito por Rzedowski (1978), en donde las plantas epífitas están representadas, sobre todo, por líquenes y musgos. En este último trabajo, hay una mención breve de epífitas vasculares (en condiciones de alta humedad atmosférica) y es probable que se refiera a algo similar a lo encontrado en el presente estudio. El mismo caso es observado en el estrato arbóreo (43 especies, $14.82 \%$ ), lo cual también es más bajo que otros BMM, pero mayor al de bosques más puros de oyamel, como el del Cerro Tláloc (11 especies, 8\%; Sánchez-González et al., 2006) y la Sierra de Zapalinamé (18 especies, 24.65\%; Encina-Domínguez et al., 2008), aunque el porcentaje en esta última es mayor, debido a la baja riqueza y diversidad. El número de plantas parásitas y saprófitas fue bajo y coincide con lo observado en otros bosques mesófilos (Alcántara y Luna, 1997, 2001; Ponce-Vargas et al., 2006; López-Pérez et al., 2011).

La considerable diversidad taxonómica del área de estudio $\left(B=210.33\right.$ especies $\left./ \mathrm{km}^{2}\right)$, permite señalar que es más diversa que los bosques citados por Sánchez-González et al. (2005) para la FVT, los cuales se desarrollan a elevaciones entre 2,500 y 3,500 m, pero es menor a la del BMM de la SMS (Lozada et al., 2003; Padilla et al., 2008; Cuadro 5). Por su parte, estudios realizados en Eloxochitlán y Tlahue- 
lompa, Hidalgo (Alcántara y Luna, 2001), muestran una riqueza menor, aun y cuando presentan una superficie tres a cinco veces mayor (Cuadro 5). En contraste, en un BMM de Minatitlán, Colima, Padilla et al. (2008) registran 248 especies de plantas vasculares, con una superficie de 194 ha (Cuadro 5).

La cantidad de helechos y plantas afines es mayor (20 especies) a la encontrada en el resto de las poblaciones o bosque de Abies de la FVT. Sin embargo, es menor a lo encontrado por Padilla et al. (2008) en Minatitlán, Colima (47 especies) y por Ponce-Vargas et al. (2006) en Lolotla, Hidalgo (32 especies). Las asteráceas por su parte, ocupan el primer lugar (46 especies, 16.15\%), pero es menor en porcentaje si se compara con los bosques puros de oyamel (Cornejo-Tenorio et al., 2003; Sánchez-González et al., 2006). La familia Orchidaceae ocupa el tercer lugar en el área de estudio (18 especies) y su riqueza es proporcionalmente mayor a la encontrada por Cornejo-Tenorio et al. (2003) en 13,551 ha de las zonas núcleo de la Reserva de la Biósfera de la Mariposa Monarca. En contraste, la localidad de Monte Grande del estado de Hidalgo (Ponce-Vargas et al., 2006) alberga 27 orquídeas, pero en una superficie tres veces mayor y un rango de variación en elevación de $530 \mathrm{~m}$ (Cuadros 2 y 5 ).

En el área de estudio se reconocen dos comunidades naturales, sus límites no son abruptos, sino graduales; sin embargo, estas se diferencían sobre todo por el cambio en la dominancia de Abies, en función del gradiente de humedad y altitud. Por su parte, en el BMM se reconocen varias asociaciones, entre ellas, la asociación Quercus-Pinus llega a ser dominante junto con otros elementos del bosque mesófilo, como Abies, Clethra, Meliosma, Symplocos, Ternstroemia y Tilia.

Debido a las diferencias florísticas puntualizadas antes y a la presencia de elementos mesófilos en el bosque de Abies en Juanacatlán, con respecto a otros bosques o poblaciones de Abies que se localizan en la FVT, se considera que el área de Juanacatlán se ajusta a lo descrito por Ohsawa (1995), como Upper Montane Cloud Forest (UMCF) para el sureste de Asia. Bajo esta denominación, se encuentra un bosque dominado por especies arbóreas micrófilas como Ilex, Symplocos y Myrsine, pero que arriba de los 2,000 m y $\operatorname{los} 20^{\circ}$ de latitud norte forman parte del sotobosque en bosques templados de coníferas, los cuales sustituyen al UMCF de acuerdo al gradiente ambiental, en su caso, bosque de Abies en los sitios fríos y húmedos. Comunidades similares constituyen, al menos en el occidente y sur de México, una faja transicional o un ensamble estructural bien definido y condicionado por la alta humedad (ver Catalán et al., 2003; Ávila et al., 2010; Cuevas-Guzmán et al., 2011).

Algunos géneros de árboles cuantitativamente importantes en los bosques mesófilos de montaña y presentes en Juanacatlán son: Alnus, Clethra, Cleyera, Cornus, Dendropanax, Fraxinus, Juglans, Meliosma, Oreopanax, Prunus,
Quercus, Styrax, Symplocos y Ternstroemia (Rzedowski, 1996). Estos géneros, además de Ilex, Myrsine, Ostrya y Tilia, se consideran diagnósticos para delimitar o definir al BMM. Además, entre las familias que Rzedowski (1996) considera que prosperan preferentemente en este tipo de comunidades, en el área de estudio se presentan las siguientes: Aquifoliaceae, Araliaceae, Begoniaceae, Clethraceae, Cornaceae, Myrsinaceae, Orchidaceae, Piperaceae, Styracaceae, Symplocaceae y Theaceae, esto es, el 33.3\%. Las familias Lauraceae y Clusiaceae con representantes preferentes o casi exclusivos de este tipo de vegetación, no se localizan en el área que se ha delimitado, pero si se encuentran dentro de la misma microcuenca a $4 \mathrm{~km}$ de nuestra área de estudio. La familia Sabiaceae, cuyos representantes mexicanos son exclusivos del bosque mesófilo de montaña también se localiza en Juanacatlán. De los géneros de plantas representados por mayor número de especies exclusivas del BMM (Rzedowski, 1996) se tiene a Begonia, Elaphoglossum, Epidendrum, Peperomia, Polypodium y Tillandsia.

El estrato arbóreo define la fisonomía y estructura de una comunidad; y puede explicar mejor la historia biogeográfica de un bosque, dados sus mecanismos de dispersión y menor capacidad de establecimiento, a diferencia de las hierbas. Para este estrato, en el área de estudio es notable la dominancia que ejercen los elementos holárticos del periodo Terciario, Mioceno tardío-Plioceno temprano, hace 5 m.a. (Graham, 1999), como Abies, Cornus, Fraxinus, Ilex, Juglans, Ostrya, Prunus y Tilia, sobre el elemento Neotropical y del este de Asia. Incluso, este último tiene algunas afinidades holárticas. Lo anterior coincide con lo aseverado por Rzedowski (1978) en torno a la elevada proporción de elementos boreales en el estrato arbóreo en estas comunidades. No obstante, este porcentaje baja en otros bosques mesófilos, como los de Chiapas, Guerrero y Oaxaca, en aquellos que tienen un amplio gradiente altitudinal. El bosque de Abies también recluta algunos elementos arbóreos de afinidad al este de Asia, como Clethra, Symplocos y Ternstroemia, caso contrario a lo que sucede con los bosques más puros de la FVT, donde casi todo el estrato arbóreo es de afinidad boreal.

Las especies Chiococca phaenostemon y Echeandia coalcomanensis son registros nuevos para Jalisco (Apéndice 1). Para la primera, Borhidi (2006) cita como su límite septentrional al estado de Guerrero por la vertiente del Pacífico, y por la del Golfo al estado de Veracruz. La segunda es señalada como una especie encontrada sólo en el extremo NO de la SMS en el estado de Michoacán (Cruden, 1987; Rodríguez-Contreras y Castro-Castro, 2005).

La distribución insular de este tipo de bosques ha producido eventos de especiación en varios taxa (Alcántara y Luna, 2001), lo que ha originado una importante proporción de elementos endémicos. En este caso, destacan los endemismos restringidos a las sierras del occidente de Jalisco (Cacoma, Cuale, La Bufa-Juanacatlán y Manantlán), 
como son Acourtia nelsonii, Astragalus jaliscensis, Cuphea baillonis, Cosmos sessilis, Dyschoriste mcvaughii, Epidendrum neogaliciense, Phaseolus perplexus, Pleurothallis sanguinolenta y Psacalium eriocarpum. No menos importante, también endémica de Jalisco pero no a esas serranías, se tiene a Salix jaliscana. Además, las especies nuevas encontradas en el área de estudio (Apéndice 1) son hasta ahora consideradas endémicas a estas sierras.

El bosque muestra buen estado de conservación y regeneración; sin embargo, dentro del área existen parches de vegetación secundaria como producto de la alteración del bosque a raíz del desarrollo de aprovechamientos forestales, incendios ocasionales, agricultura de temporal y pastoreo. Se caracteriza por la presencia de especies exóticas y ruderales, como Avena sativa, Brassica rapa, Lepidium virginicum, Anagallis arvensis, entre otras.

Conservación del bosque de Abies. Esta comunidad se encuentra dentro del polígono del Área de Protección de Recursos Naturales de la Cuenca Alimentadora del Distrito de Riego 043, fracción Sierra de Vallejo-Río Ameca (CONANP, 2014) y en la Región Terrestre Prioritaria 62 Sierra de Vallejo-Río Ameca (Arriaga et al., 2000). Alberga cinco especies leñosas bajo alguna categoría de protección otorgada por la la NOM-059-SEMARNAT-2010. Lo que resulta sorprendente es el desconocimiento por parte de la Comisión Natural de Áreas Naturales Protegidas, hasta el año 2011, de la existencia del bosque de Abies. Por lo que se recomiendan algunas medidas de conservación: (1) Asegurar la permanencia del oyamel de acuerdo a la categoría de protección que se le designa en la NOM-059-SEMARNAT-2010. Se debe contemplar la realización de monitoreos regulares que eviten la tala clandestina, así como la difusión y fortalecimiento de estrategias de uso sustentable de los recursos del bosque, dirigido hacia sus propietarios y habitantes de las poblaciones cercanas (Cimarrón, Juanacatlán, La Yerbabuena, Navidad). (2) Prevenir y llevar un control adecuado de incendios. (3) Desarrollar estudios estructurales y demográficos de la población de oyamel y especies arbóreas asociadas, y relacionar estos datos con variables ambientales que permitan un mejor entendimiento del funcionamiento del bosque. (4) Promover y evaluar la población de oyamel como una prioridad para la conservación, tanto a nivel estatal como federal, justificado por la alta y particular diversidad florística que manifiesta. Esto implicaría el desarrollo de inventarios biológicos de otros grupos, tales como hongos y animales.

Es importante recordar que las masas forestales de oyamel, destacan por la captación de agua y regulación de la escorrentía (Sánchez-González et al., 2006), por lo que el deterioro ambiental en este tipo de comunidades puede acarrear pérdida de agua que afecte el balance hidrológico y abastecimiento de zonas urbanas, en este caso la más afectada pudiera ser Mascota. Bajo estas circunstancias, es necesario el monitoreo continuo que garantice la generación de nuevas alternativas para su conservación.

\section{Agradecimientos}

Se agradece al personal del herbario IBUG las facilidades otorgadas para la consulta de la colección y por la identificación de materiales a Martha Cedano-Maldonado (helechos y afines), Mollie Harker (Asteraceae), Guadalupe MunguíaLino (Iridaceae), Esteban Alberto Suarez-Muro (Fabaceae) y Aarón Rodríguez-Contreras (Solanaceae y monocotiledóneas petaloides). Asimismo, agradecemos a Jaqueline Reynoso-Dueñas, Jorge Alberto Pérez de la Rosa y Miguel Ángel Macías-Rodríguez por la revisión crítica a una versión preliminar del manuscrito y a los revisores anónimos por las importantes sugerencias y correcciones. ACC agradece a la fundación Idea Wild por el apoyo otorgado. Este trabajo no hubiera sido posible sin la compañía, el consejo e intenso trabajo de campo realizado por Ignacio Guerrero, Luis Fernando-Colín y Ernesto De Castro-Arce. De manera especial agradecemos al pueblo de Juanacatlán y Navidad la hospitalidad otorgada. Agradecemos al Ing. Luis Felipe Bugarin (GEOServicios) la elaboración de la cartografía.

\section{Literatura citada}

Alcántara A.O. y Luna V.I. 1997. Florística y análisis biogeográfico del bosque mesófilo de montaña de Tenango de Doria, Hidalgo, México. Anales del Instituto de Biología, Serie Botánica 68:57-106.

Alcántara A.O. y Luna V.I. 2001. Análisis florístico de dos áreas con bosque mesófilo de montaña en el estado de Hidalgo, México: Eloxochitlán y Tlahuelompa. Acta Botanica Mexicana 54:51-87.

Almeida-Leñero G.L. 1997. Vegetación, fitogeografía y paleoecología del zacatonal alpino y bosques montanos de la región central de México. Tesis doctoral, Laboratorio Hugo de Vries, Departamento de Palinología y Paleo/Actuo-Ecología, Universidad de Ámsterdam, Ámsterdam. 255 pp.

Álvarez del Castillo C. 1987. La Vegetación de la Sierra del Ajusco. Departamento de Prehistoria, Instituto Nacional de Antropología e Historia, Cuaderno de Trabajo 33, México, D.F.

APG III. Angiosperm Phylogeny Group. 2009. An update of the angiosperm phylogeny group classification for the orders and families of flowering plants: APG III. Botanical Journal of the Linnean Society 161:105-121.

Arriaga L., Espinoza J.M., Aguilar C., Martínez E., Gómez L. y Loa E. Coords. 2000. Regiones Terrestres Prioritarias de México. Comisión Nacional para el Conocimiento y uso de la Biodiversidad. México, D.F. <http://www.conabio.gob.mx/conoci miento/regionalizacion/doctos/terrestres.html> (consultado 7 de enero 2013).

Ávila S.P., Sánchez-González A. y Catalán E.C. 2010. Estructura y composición de la vegetación del Cañón del Zopilote, Guerrero, México. Revista Chapingo. Serie Ciencias Forestales y del Ambiente 16:119-138.

Borhidi A. 2006. Rubiáceas de México. Akadémiai Kiadó, Budapest. 
Boyas D.J.C. 1993. Flora fanerogámica del Campo Experimental Forestal "San Juan Tetla", Puebla. Folleto Técnico no. 114. 2 a. ed. Secretaría de Agricultura y Recursos Hidráulicos, Instituto de Investigaciones Forestales y Agropecuarias, División Forestal, México, D.F.

Briones V.O.L. 1991. Sobre la flora, vegetación y fitogeografía de la Sierra de San Carlos, Tamaulipas. Acta Botanica Mexicana 16:15-43.

Calderón de Rzedowski G. 1991. Papaveraceae. Flora del Bajío y Regiones Adyacentes 1:1-36.

Calderón de Rzedowski G. 1992. Cistaceae. Flora del Bajío y Regiones Adyacentes 2:1-27.

Calderón de Rzedowski G. y Rzedowski J. 2001. Flora fanerogámica del Valle de México. 2a ed. Instituto de Ecología, A.C., Comisión Nacional para el Conocimiento y Uso de la Biodiversidad, Pátzcuaro.

Carranza E. 2007. Convolvulaceae. Flora del Bajío y Regiones Adyacentes 151:1-129.

Carranza G.E. 2008. Styracaceae. Flora de Jalisco y Áreas Colindantes 22:1-14.

Castillejos-Cruz C. y Ramírez-Ramírez R.I. 1992. Florística y vegetación del estado de Tlaxcala. Tesis licenciatura, Facultad de Estudios Superiores Zaragoza, Universidad Nacional Autónoma de México, México, D.F. 88 pp.

Castillejos-Cruz C. y Solano E. 2008. Polygonaceae. Flora del Bajío y Regiones Adyacentes 153:1-49.

Catalán H.C., López-Mata L. y Terrazas T. 2003. Estructura, composición florística y diversidad de especies leñosas de un bosque mesófilo de montaña de Guerrero, México. Anales del Instituto de Biología, Serie Botánica 74:209-230.

CEA. Comisión Estatal del Agua de Jalisco. 2012. Sistema de Información del Agua <http://www.ceajalisco.gob.mx/cuencas/ ameca-ixt.html> (consultado 7 de enero 2012).

CETENAL. Comisión de Estudios del Territorio Nacional. 1974. Carta geológica. Mascota. F13D61. Escala 1:50 000.

CETENAL. Comisión de Estudios del Territorio Nacional. 1975. Carta edafológica. Mascota. F13D61. Escala 1:50 000.

Christenhusz M.J.M., Reveal J.L., Farjon A., Gardner M.F., Mill R.R. y Chase M.W. 2011a. A new classification and linear sequence of extant gymnosperms. Phytotaxa 19:55-70.

Christenhusz M.J.M., Zhang X.C. y Schneider H. 2011b. A linear sequence of extant families and genera of lycophytes and ferns. Phytotaxa 19:7-54.

Cisneros-Lepe E.A. 2005. Estructura, composición florística y diversidad de los bosques de Abies en el estado de Jalisco, México. Tesis licenciatura, Universidad de Guadalajara, Guadalajara. $101 \mathrm{pp}$.

CONANP. Comisión Nacional de Áreas Naturales Protegidas. 2006. Programa de Conservación y Manejo Parque Nacional Volcán Nevado de Colima. Comisión Nacional de Áreas Naturales Protegidas, Salinas Impresoras, México, D. F.

CONANP. Comisión Nacional de Áreas Naturales Protegidas. 2014. Cuenca alimentadora del Distrito de Riego 043 estado de Nayarit, en lo respectivo a las subcuencas de los ríos Ameca, Atenguillo, Bolaños, Grande de Santiago Juchipila, Atengo y Tlaltenango. <https://simec.conanp.gob.mx/Info_comple ta_ext.php?id_direccion=198> (consultado 1 de septiembre de 2014)

Cornejo-Tenorio G., Casas A., Farfán B., Villaseñor J.L. e IbarraManríquez G. 2003. Flora y vegetación de las zonas núcleo de la reserva de la biosfera Mariposa Monarca, México. Boletín de la Sociedad Botánica de México 73:43-62.

Cruden R.W. 1987. New species of Echeandia (Liliaceae) from Nueva Galicia. Contributions from the University of Michigan Herbarium 16:129-133.

Cuevas-Guzmán R., Cisneros-Lepe E.A., Jardel-Peláez E.J., Sánchez-Rodríguez E.V., Guzmán-Hernández L., Núñez-López N.M. y Rodríguez-Guerrero C. 2011. Análisis estructural y de diversidad en los bosques de Abies de Jalisco, México. Revista Mexicana de Biodiversidad 82:1219-1233.

Encina-Domínguez J.A., Encina-Domínguez F.J., Mata-Rocha E. y Valdés-Reyna J. 2008. Aspectos estructurales, composición florística y caracterización ecológica del bosque de oyamel de la Sierra de Zapalinamé, Coahuila, México. Boletín de la Sociedad Botánica de México 83:13-24.

Fernández N.R. 1996. Rhamnaceae. Flora del Bajío y Regiones Adyacentes 43:1-68.

Ferrusquía-Villafranca I. 1998. Geología de México: una sinopsis. En: Ramamoorthy T.P., Bye R., Lot A. y Fa J. Eds. Diversidad Biológica de México: Orígenes y Distribución, pp. 3-108, Instituto de Biología, Universidad Nacional Autónoma de México, México, D.F.

Figueroa R.B.L. y Moreno G.S. 1993. Estructura y sucesión en poblaciones de Abies religiosa (H.B.K.) Schlecht. \& Cham. y Abies religiosa var. emarginata Loock et Martínez ex Martínez, en la Sierra de Manantlán, Jalisco. Agrociencia, Serie Recursos Naturales Renovables 3:49-63.

Font Quer P. 1953. Diccionario de Botánica. Editorial Labor, Barcelona.

Frías-Castro A., Castro-Castro A., González-Gallegos J.G., Suárez-Muro E.A. y Rendón-Sandoval F.J. 2013. Flora vascular y vegetación del Cerro El Tepopote, Jalisco, México. Botanical Sciences 91:53-74.

García-Arévalo A. 2008. Vegetación y Flora de un bosque relictual de Picea chihuahuana Martínez del Norte de México. Polibotánica 25:45-68.

García E. 2004. Modificaciones al Sistema de Clasificación Climática de Köppen. $5^{\text {a }}$ ed. Instituto de Geografía, Universidad Nacional Autónoma de México, México, D.F.

García R.M. 1993. Vegetación. En: Luna V.I. y Llorente B.J. Eds. Historia Natural del Parque Ecológico Estatal Omiltemi, Chilpancingo, Guerrero, México, pp 39-55, Comisión Nacional para el Conocimiento y Uso de la Biodiversidad, Universidad Nacional Autónoma de México, México, D.F.

García-Peña M.R. y González-Gallegos J.G. 2013. Cunila jalisca$n a$ (Lamiaceae) a new species from Jalisco. Mexico. Phytotaxa 125:17-24.

Gentry A.H. 1982. Neotropical floristic diversity: phytogeographical connections between Central and South America, Pleistocene climatic fluctuations, or and accidente of the Andean orogeny? Annals of the Missouri Botanical Garden 69: 557593.

Gómez-Tuena A., Orozco-Esquivel M.T. y Ferrari L. 2005. Petrogénesis ígnea de la Faja Volcánica Transmexicana. Boletín de la Sociedad Geológica Mexicana 3:227-283.

González-Villarreal L.M. 1986. Contribución al conocimiento del género Quercus (Fagaceae) en el estado de Jalisco, México. Colección Flora de Jalisco 1:1-240.

González-Villarreal L.M. 1990. Las Ericáceas de Jalisco, México. Colección Flora de Jalisco. 2:1-140. 
González-Villarreal L.M. 1996a. La familia Clethraceae en el estado de Jalisco, México. Colección Flora de Jalisco 5:1-35.

González-Villarreal L. M. 1996b. La familia Cornaceae en el estado de Jalisco, México. Colección Flora de Jalisco 4:1-18.

González-Villarreal L.M. 2000a. La familia Aquifoliaceae en el estado de Jalisco, México. Colección Flora de Jalisco 6:1-26.

González-Villarreal L.M. 2000b. La familia Betulaceae en el estado de Jalisco, México. Colección Flora de Jalisco 8:1-40.

González-Villarreal L.M. 2000c. La familia Garryaceae en el estado de Jalisco, México. Colección Flora de Jalisco 7:1-25.

González-Villarreal L.M. 2001. La familia Theaceae en el estado de Jalisco, México. Colección Flora de Jalisco 12:1-38.

González-Villarreal L.M. 2002. La familia Symplocaceae en el estado de Jalisco, México. Colección Flora de Jalisco 13:1-31.

Graham A. 1999. The tertiary history of the northern temperate element in the northern Latin American biota. American Journal of Botany 86:32-38.

Graham S.A. 1994. Lythraceae. Flora del Bajío y Regiones Adyacentes 24:1-62.

Guerrero-Hernández R. 2012. Flora y vegetación asociada a Abies guatemalensis var. jaliscana Martínez en Juanacatlán, municipio de Mascota, Jalisco. Tesis de Licenciatura, Universidad de Guadalajara, Guadalajara. 62 pp.

Hernández-López L. 1995. The endemic flora of Jalisco, Mexico. Centers of endemism and implications for conservation. Tesis maestría, Universidad de Wisconsin, Madison, 74 pp.

Hernández-Rosales M.R.A. 1995. Estudio florístico fanerogámico del Parque Nacional El Chico, estado de Hidalgo. Tesis licenciatura, Escuela Nacional de Estudios Profesionales Iztacala, Universidad Nacional Autónoma de México, Tlalnepantla. 73 pp.

Hijmans R.J., Cameron S.E., Parra J.L., Jones P.G. y Jarvis A. 2005. Very high resolution interpolated climate surfaces for global land areas. International Journal of Climatology 25:19651978.

Holt B.G., Lessard J.P., Borregaard M.K., Fritz S.A., Araújo M.B., Dimitrov D., Fabre P.H., Graham C.H., Graves G.R., Jønsson K.A., Nogués-Bravo D., Wang Z., Whittaker R.J., Fjeldså J., Rahbek C. 2013. An update of Wallace's zoogeographic regions of the world. Science 339:74-78.

Humphries C.J., Vane-Wright R.I. y Williams P.H. 1991. Biodiversity reserves: setting new priorities for the conservation of the wildlife. Park 2:34-38.

IPNI. International Plant Names Index. 2013. The International Plant Names Index. <www.ipni.org> (consultado septiembre y octubre 2013).

Jaramillo-Correa J.P., Aguirre-Planter E., Khasa D.P., Eguiarte L.E., Piñero D., Furnier G.R. y Bousquet J. 2008. Ancestry and divergence of subtropical montane forest isolates: molecular biogeography of the genus Abies (Pinaceae) in southern México and Guatemala. Molecular Ecology 17:2476-2490.

Jiménez R.J., ContrerasJ.J.L., González F.R.E., Antonio O.R., Lozano V.G. y TorresR.S. 1993. Plantas Vasculares. En: Luna V.I. y Llorente B.J. Eds. Historia Natural del Parque Ecológico Estatal Omiltemi, Chilpancingo, Guerrero, México, pp. 127-250, Comisión Nacional para el Conocimiento y Uso de la Biodiversidad, Universidad Nacional Autónoma de México, México, D.F.

López-Pérez Y., Tejero-Díez J., Torres-Díaz A.N. y Luna-Vega I. 2011. Flora del bosque mesófilo de montaña y vegetación adyacente en Avándaro, Valle de Bravo, Estado de México, México. Boletín de la Sociedad Botánica de México 88:35-53.
Lot A. y Chiang C.F. 1986. Manual de Herbario. Consejo Nacional de la Flora de México, A. C., México, D.F.

Lozada L., León M.E., Rojas J. y Santiago R. 2003. Bosque mesófilo de montaña en el Molote. Estudios Florísticos en Guerrero 13:1-35.

Luna I., Almeida L., Villers L. y Lorenzo L. 1988. Reconocimiento florístico y consideraciones fitogeográficas del bosque mesófilo de montaña de Teocelo, Veracruz. Boletín de la Sociedad Botánica de México 48:35-63

Luna I., Almeida-Leñero L. y Llorente-Bousquets J. 1989. Florística y aspectos fitogeográficos del bosque mesófilo de montaña de las cañadas de Ocuilan, Estados de Morelos y México. Anales del Instituto de Biología, Universidad Nacional Autónoma de México, Serie Botánica 59:63-87.

Luquín S.H., Nieves H.G. y Ayala R.J.M. 2004. Vegetación del norte de Jalisco. En: Vázquez-García J.A., Cházaro B.M.J., Nieves H.G., Vargas-Rodríguez Y.L., Vázquez G.M. y Flores M.A. Eds. Flora del Norte de Jalisco y Etnobotánica Huichola, pp. 77-83, Centro Universitario de Ciencias Biológicas y Agropecuarias, Centro Universitario de Ciencias Sociales y Humanidades de la Universidad de Guadalajara, Guadalajara.

Madrigal-Sánchez X. 1967. Contribución al conocimiento de la ecología de los bosques de oyamel (Abies religiosa (H.B.K.) Schl. \& Cham.) en el valle de México. Boletín Técnico No. 18. Instituto Nacional de Investigaciones Forestales, México D.F.

Manzanilla H. 1974. Investigaciones Epidométricas y Silvícolas en Bosques Mexicanos de Abies religiosa. Dirección General de Información y Relaciones Públicas de la Secretaría de Agricultura y Ganadería, México, D.F.

May-Nah A. 1971. Estudio fitoecológico del Campo Experimental San Juan Tetla, estado de Puebla, México. Tesis licenciatura, Escuela Nacional de Ciencias Biológicas, Instituto Politécnico Nacional, México, D.F. 130 pp.

McVaugh R. 1961. Euphorbiaceae novae Novo-Galicianae. Brittonia 13:145-205.

McVaugh R. 1984. Compositae. Flora Novo-Galiciana 12. University of Michigan Press, Ann Arbor.

McVaugh R. 1985. Orchidaceae. Flora Novo-Galiciana 16. University of Michigan Press, Ann Arbor.

McVaugh R. 1987. Leguminosae. Flora Novo-Galiciana 5. University of Michigan Press, Ann Arbor.

McVaugh R. 1989. Bromeliaceae to Dioscoreaceae. Flora NovoGaliciana 15. University of Michigan Press, Ann Arbor.

McVaugh R. 1992. Gymnosperms and Pteridophytes. Flora NovoGaliciana 17. University of Michigan Press, Ann Arbor.

Meave J., Soto A.M.A., Calvo I.L.M., Paz H.H. y ValenciaA. S. 1992. Análisis sinecológico del bosque mesófilo de montaña de Omiltemi, Guerrero. Boletín de la Sociedad Botánica de México 52:31-77.

Mickel J.T. y Smith A.R. 2004. The Pteridophytes of Mexico. Memoirs of the New York Botanical Garden 88:1-1054.

Moreno N.P. 1984. Glosario Botánico Ilustrado. Instituto Nacional de Investigaciones sobre Recursos Bióticos, C.E.C.S.A., Xalapa.

Morrone J.J. 2005. Hacia una síntesis biogeográfica de México. Revista Mexicana de Biodiversidad 76:207-252.

Narave F.H. 1985. La vegetación del Cofre de Perote, Veracruz, México. Biotica 10:35-64.

Nieto P.P.C. 1995. Estudio sinecológico del bosque de oyamel de la cañada de Contreras, Distrito Federal. Revista Ciencia Forestal en México 20:3-34. 
Ocampo A.G. 2003. Plantaginaceae. Flora del Bajío y Regiones Adyacentes 120:1-21.

Ohsawa M. 1995. The montane cloud forest and its gradational changes in southeast Asia. En: Hamilton L.S., Juvik J.O. y Scatena F.N. Eds. Tropical Montane Cloud Forests, pp. 254-265, Springer-Verlag, Nueva York.

Osorio-Rosales M.L. 1984. Flora y vegetación de la parte superior de la Sierra de Monte Alto en el Valle de México. Tesis licenciatura, Facultad de Ciencias, Universidad Nacional Autónoma de México, México, D.F. 109 pp.

Padilla V.E., Cuevas G.R. y Koch S.D. 2008. Plantas vasculares y vegetación de la parte alta del arroyo Agua Fría, municipio de Minatitlán, Colima, México. Acta Botanica Mexicana 84:25-72.

Patiño S.A. 2001. Boraginaceae. En: Calderón de Rzedowski G. y Rzedowski J. Eds. Flora Fanerogámica del Valle de México. 2a. ed., pp. 603-614, Instituto de Ecología, A.C., Comisión Nacional para el Conocimiento y Uso de la Biodiversidad, Pátzcuaro.

Pérez-Calix E. 2009. Oxalidaceae. Flora del Bajío y Regiones Adyacentes 164:1-49.

Pérez-Calix E. y Carranza G.E. 2005. Hydrophyllaceae. Flora del Bajío y Regiones Adyacentes 139:1-48.

Pérez de la Rosa J.A. 1987. Notas del herbario del Instituto de Botánica de la Universidad de Guadalajara (IBUG): primer reporte de Abies durangensis Martínez, en Jalisco. Tiempos de Ciencia 8:37-39.

Ponce-Vargas A., Luna-Vega I., Alcántara-Ayala O. y Ruiz-Jiménez C.A. 2006. Florística del bosque mesófilo de montaña de Monte Grande, Lolotla, Hidalgo, México. Revista Mexicana de Biodiversidad 77:177-190.

PRODEFO. Programa de Desarrollo Forestal de Jalisco. 1999. Superficies de recursos forestales del estado de Jalisco: Según región COPLADE, municipio y área natural de conservación). Programa de Desarrollo Forestal de Jalisco, Guadalajara.

Quintana-Ascencio P.F. y Gonzalez-Espinosa M. 1993. Afinidad fitogeográfica y papel sucesional de la flora leñosa de los bosques de pino-encino de los Altos de Chiapas, México. Acta Botanica Mexicana 21:43-57.

Ramírez-Delgadillo R., Vargas-Ponce O., Arreola-Nava H.J., Cedano-Maldonado M., González-Tamayo R., González-Villarreal L.M., Harker M., Hernández-López L., Martínez-González R.E., Pérez de la Rosa J.A., Rodríguez-Contreras A., Reynoso-Dueñas J.J., Villarreal de Puga L.M. y Villaseñor-Ríos J.L. 2010. Catálogo de Plantas Vasculares de Jalisco. Universidad de Guadalajara, Sociedad Botánica de México, Universidad Autónoma Metropolitana, Guadalajara.

Ramos-Herrera M.J. 1991. Estudio de la distribución del género Abies en la Sierra de Manantlán, Jalisco. Tesis licenciatura, Universidad de Guadalajara, Guadalajara, Jalisco. 102 pp.

Reynoso-Dueñas J.J., Hernández L.L., Ramírez-Delgadillo R., Harker M., Cedano M.M. y Álvarez B.I.L. 2006. Catálogo preliminar de la flora vascular y micobiota del municipio de San Sebastián del Oeste, Jalisco, México. Ibugana 14:51-91.

Rodríguez-Contreras A. y Castro-Castro A. 2005. Notas sobre Echeandia pihuamensis, E. robusta y E. sinaloensis y estado de conservación de las especies mexicanas. Ibugana 13:3-9.

Rzedowski J. 1978. Vegetación de México. Editorial Limusa, México, D.F.

Rzedowski J. 1991. Diversidad y orígenes de la flora fanerogámica de México. Acta Botanica Mexicana 14:3-21.
Rzedowski J. 1996. Análisis preliminar de la flora vascular de los bosques mesófilos de montaña de México. Acta Botanica Mexicana 35:25-44.

Rzedowski J. y Calderón de Rzedowski G. 1995. Geraniaceae. Flora del Bajío y Regiones Adyacentes 40:1-37.

Rzedowski J. y Calderón de Rzedowski G. 2004. Oleaceae. Flora del Bajio y Regiones Adyacentes 124:1-37.

Rzedowski J. y McVaugh R. 1966. La vegetación de Nueva Galicia. University of Michigan Press, Ann Arbor.

Sánchez-González A. 2004. Análisis sinecológico, florístico y biogeográfico de la vegetación del norte de la Sierra Nevada. Tesis doctorado en Ciencias (Botánica), Colegio de Postgraduados, Montecillo. 153 pp.

Sánchez-González A., López-Mata L. y Granados-Sánchez D. 2005. Semejanza florística entre los bosques de Abies religiosa (H.B.K.) Cham. \& Schltdl. de la Faja Volcánica Transmexicana. Investigaciones Geográficas, Boletín del Instituto de Geografía UNAM 56:62-76.

Sánchez-González A., López-Mata L. y Vibrans H. 2006. Composición y patrones de distribución geográfica de la flora del bosque de oyamel del cerro Tláloc, México. Boletín de la Sociedad Botánica de México 79:67-78.

SEMARNAT. Secretaría de Medio Ambiente y Recursos Naturales. 2010. Norma Oficial Mexicana NOM-059-SEMARNAT2010. Protección ambiental-Especies nativas de México de flora y fauna silvestres-Categorías de riesgo y especificaciones para su inclusión, exclusión o cambio-Lista de especies en riesgo. <dof.gob.mx/nota_detalle.php?codigo $=5173091 \&$ fecha $=3$ 0/12/2010> (consultado 7 de enero 2013)

SPP. Secretaría de Programación y Presupuesto. 1980. Carta de climas. Escala 1:1 000 000. Hoja Guadalajara. Coordinación General de los Servicios Nacionales de Estadística, Geografía e Informática; Dirección General de Geografía del Territorio Nacional. México, D.F.

SPP. Secretaría de Programación y Presupuesto. 1981. Síntesis geográfica del estado de Jalisco. Coordinación General de los Servicios Nacionales de Estadística, Geografía e Informática; Dirección General de Geografía del Territorio Nacional. México, D. F.

Squeo F.A., Cavieres L.A., Arancio G., Novoa J.E., Matthei O., Marticorena C., Rodríguez R., Arroyo M.T.K. y Muñoz M. 1998. Biodiversidad de la flora vascular en la Región de Antofagasta, Chile. Revista Chilena de Historia Natural 71:571591.

Thiers B. 2011. Index Herbariorum. New York Botanical Garden's Virtual Herbarium. <sweetgum.nybg.org/ih/> (consultado 7 de enero 2013).

Tropicos. 2011. Tropicos.org. Missouri Botanical Garden. <www. tropicos.org> (consultado de enero-octubre 2013).

Vázquez G.J.A., Reynoso D.J.J., Vargas R.Y.L. y Frías U.H.G. 2000. Jalisco Costa-Norte: Patrimonio Ecológico, Cultural y Productivo de México. Universidad de Guadalajara, Guadalajara.

Vázquez-García J.A., Vargas R.Y.L. y Aragón F. 2000. Descubrimiento de un bosque de Acer-Podocarpus-Abies en el municipio de Talpa de Allende, Jalisco, México. Boletín del Instituto de Botánica de la Universidad de Guadalajara 7:159-183.

Vega-Aviña R. 1982. Manual de la flora de la estación experimental de enseñanza e investigación y servicios forestales Zoquiapan. Tesis maestría en Ciencias, Colegio de Postgraduados, Chapingo. 364 pp. 
Velázquez A. y Cleef A.M. 1993. The plant communities of the volcanoes Tlaloc and Pelado, Mexico. Phytocoenologia 22:145191.

Villarreal Q.J.A. 2000. Caprifoliaceae. Flora del Bajío y de Regiones Adyacentes 88:1-17.

Villarreal-Q. J.A. y Estrada-C. E.2011. El género Gentianopsis (Gentianaceae) en México. Polibotánica 32:1-7.

Villaseñor J.L. 2003. Diversidad y distribución de las Magnolio- phyta de México. Interciencia 28:160-167.

Villaseñor J.L. 2004. Los géneros de plantas vasculares de la flora de México. Boletín de la Sociedad Botánica de México 75:105135.

Villaseñor J.L. 2010. El Bosque Húmedo de Montaña en México y sus Plantas Vasculares: Catálogo Florístico-Taxonómico. Comisión Nacional para el Conocimiento y Uso de la Biodiversidad, Universidad Nacional Autónoma de México, México, D.F.

Recibido: 22 de agosto 2013

Aceptado: 8 de noviembre de 2013 
Apéndice 1. Lista de plantas vasculares de un bosque de Abies y el bosque mesófilo de montaña adyacente en Juanacatlán, Mascota, Jalisco. Abreviaturas. Formas biológicas (FB) por hábito: A, árbol; a, arbusto; B, bejuco; H, hierba; hábitat: T, terrestre; E, epífito; R, rupícola; y tipo de nutrición: $U$, autótrofo; $\mathrm{P}$, hemiparásito; $\mathrm{S}$, saprófito. Tipos de vegetación (TV): BA, bosque de Abies; BMM, bosque mesófilo de montaña. Colectores (C): AC, Arturo Castro Castro; FC, Fernando Colín Nolasco; JG, Jesús González Gallegos; RR, Raymundo Ramírez Delgadillo; RG, Ricardo Guerrero Hernández. Símbolos: *taxa endémicos de México; **taxa endémicos de Jalisco; ${ }^{* * *}$ especies nuevas; $* * *$ nuevos registros para la flora de Jalisco.

\begin{tabular}{llllll}
\hline TAXA & FB & TV & C
\end{tabular}

\section{HELECHOS}

\section{SUBCLASE EQUISETIDAE}

EQUISETACEAE

Equisetum hyemale subsp. affine (Engelm.) Calder \& Roy L.Taylor HTU

BMM

BA

RG 359

\section{SUBCLASE OPHIOGLOSSIDAE \\ OPHIOGLOSSACEAE \\ Botrychium virginianum (L.) Sw.}

\section{SUBCLASE POLYPODIIDAE}

ASPLENIACEAE

Asplenium cuspidatum Lam.

Asplenium monanthes L.

DENNSTAEDTIACEAE

Pteridium aquilinum var. feei (W.Schaffn. ex Fée) J.A.Thomson,

Michel \& Mehltr.

DRYOPTERIDACEAE

Elaphoglossum rzedowskii Mickel*

POLYPODIACEAE

Phlebodium areolatum (Willd.) J.Sm.

Pleopeltis angusta Humb. \& Bonpl. ex Willd.

Pleopeltis polylepis (Roem. ex Kunze) T.Moore*

Polypodium madrense J.Sm.*

Polypodium martensii Mett.*

Polypodium platylepis Mett. ex Kuhn

Polypodium polypodioides var. aciculare Weath.

Polypodium sanctae-rosae (Maxon) C.Chr.

PTERIDACEAE

Adiantum andicola Liebm.

Cheilanthes farinosa (Forssk.) Kaulf.

Cheilanthes hirsuta Link

Pteris cretica L.

THELYPTERIDACEAE

Thelypteris cheilanthoides (Kunze) Proctor

Thelypteris rudis (Kunze) Proctor

$\begin{array}{llll}\text { HE(R)U } & \text { BMM } & \text { BA } & \text { RG } 307 \\ \text { HEU } & \text { BMM } & \text { BA } & \text { RG } 296 \\ \text { HTU } & \text { BMM } & \text { BA } & \text { RG } 85 \\ & & & \\ \text { HTU } & \text { BMM } & & \text { RG } 454 \\ \text { HE(R)U } & \text { BMM } & & \text { RG } 421 \\ \text { HEU } & \text { BMM } & & \text { RG } 298 \\ \text { HEU } & \text { BMM } & \text { BA } & \text { RG } 371 \\ \text { HE(T)U } & \text { BMM } & \text { BA } & \text { RG } 423 \\ \text { HEU } & \text { BMM } & & \text { RG } 455 \\ \text { HEU } & \text { BMM } & & \text { RG } 99 \\ \text { HRU } & \text { BMM } & & \text { RG } 361 \\ \text { HEU } & \text { BMM } & & \text { RG } 301 \\ \text { HTU } & \text { BMM } & \text { BA } & \text { RG } 235 \\ \text { HTU } & \text { BMM } & & \text { RG } 467 \\ \text { HTU } & \text { BMM } & & \text { RG } 430 \\ \text { HTU } & \text { BMM } & & \text { FC } 471 \\ \text { HTU } & \text { BMM } & & \text { RG } 306 \\ \text { HTU } & \text { BMM } & \text { BA } & \text { RG } 417\end{array}$

\section{GIMNOSPERMAS}

\section{SUBCLASE PINIDAE}

PINACEAE

Abies flinckii Rushforth

$\begin{array}{llll}\text { ATU } & \text { BMM } & \text { BA } & \text { RG } 641 \\ \text { ATU } & \text { BMM } & \text { BA } & \text { RG 521 } \\ \text { ATU } & \text { BMM } & \text { BA } & \text { RG 520 } \\ \text { ATU } & \text { BMM } & \text { BA } & \text { RG 32 } \\ \text { ATU } & & \text { BA } & \text { RG 355 } \\ \text { ATU } & \text { BMM } & \text { BA } & \text { RG 521 }\end{array}$


Apéndice 1. Continuación

TAXA

ANGIOSPERMAS

Magnolídeas

PIPERACEAE

Peperomia campylotropa A.W.Hill.

Peperomia galioides Kunth

\section{Monocotiledóneas}

ASPARAGACEAE

Agave maximiliana Baker

Agave sp.

Allium kunthii G.Don

Echeandia coalcomanensis Cruden*, ****

Echeandia imbricata Cruden*

Furcraea parmentieri (Roelz ex Ortgies) García-Mend.*

Prochnyanthes sp. nov. ined.***

AMARYLLIDACEAE

Sprekelia formosissima (L.) Herb.

BROMELIACEAE

Tillandsia bourgaei Baker*

Tillandsia calothyrsus Mez*

CYPERACEAE

Carex turbinata Liebm.*

Cyperus esculentus L.

Cyperus aff. hermaphroditus (Jacq.) Standl.

Cyperus sp.

Kyllinga pumila Michx.

Rhynchospora aristata var. suberecta Kük.*

COMMELINACEAE

Commelina coelestis Willd.

Gibasis linearis (Benth.) Rohweder*

Tradescantia commelinoides Schult. \& Schult. f.

Tradescantia crassifolia Cav.

DIOSCOREACEAE

Dioscorea minima B.L.Rob. \& Seaton*

HYPOXIDACEAE

Hypoxis lucens McVaugh*

IRIDACEAE

Sisyrinchium cernuum (E.P.Bicknell) Kearney

Sisyrinchium convolutum Nocca

Sisyrinchium palmeri Greenm.*

Tigridia multiflora (Baker) Ravenna*

Tigridia suarezii Aarón Rodr. \& Ortiz-Cat.

ORCHIDACEAE

Bletia purpurata A.Rich. \& Galeotti

Dichromanthus aurantiacus (La Llave \& Lex.) Salazar \& Soto Arenas

Encyclia sp.

Epidendrum neogaliciense Hágsater \& R.González**

Govenia liliacea (La Llave \& Lex.) Lindl.

Govenia superba (La Llave \& Lex.) Lindl.

Habenaria flexuosa Lindl.
FB

TV

C

HTU

BMM

BMM

RG 364

HEU

RG 442, 456

\begin{tabular}{|c|c|c|c|}
\hline aTU & BMM & $\mathrm{BA}$ & RG 642 \\
\hline aTU & BMM & $\mathrm{BA}$ & RG 643 \\
\hline HTU & & $\mathrm{BA}$ & RG 305 \\
\hline HTU & BMM & $\mathrm{BA}$ & AC 2469; RG 357 \\
\hline HTU & $\mathrm{BMM}$ & & RG 360 \\
\hline aTU & & $\mathrm{BA}$ & RG 644 \\
\hline HTU & BMM & & AC 2500; RG 413 \\
\hline HTU & & $\mathrm{BA}$ & RG 322 \\
\hline HEU & BMM & & RG 258 \\
\hline HEU & BMM & & RG 281 \\
\hline HTU & & $\mathrm{BA}$ & RG 398, 401 \\
\hline HTU & & $\mathrm{BA}$ & RG 378 \\
\hline HTU & & $\mathrm{BA}$ & RG 377 \\
\hline HTU & & $\mathrm{BA}$ & RG 386 \\
\hline HTU & BMM & $\mathrm{BA}$ & RG 403 \\
\hline HTU & $\mathrm{BMM}$ & & RG 430 \\
\hline HTU & BMM & $\mathrm{BA}$ & RG 96 \\
\hline HTU & BMM & $\mathrm{BA}$ & RG 356 \\
\hline HTU & BMM & & RG 293 \\
\hline HTU & $\mathrm{BMM}$ & $\mathrm{BA}$ & RG 437 \\
\hline HTU & BMM & & RG 440 \\
\hline HTU & & $\mathrm{BA}$ & RG 374 \\
\hline HTU & & $\mathrm{BA}$ & RG 561 \\
\hline HTU & $\mathrm{BMM}$ & & RG 339 \\
\hline HTU & BMM & $\mathrm{BA}$ & RG 441 \\
\hline HTU & BMM & $\mathrm{BA}$ & RG 411 \\
\hline HTU & BMM & & RG 461 \\
\hline HTU & BMM & & RG 512 \\
\hline HTU & & $\mathrm{BA}$ & RG 365 \\
\hline HEU & BMM & & RG 645 \\
\hline HEU & BMM & & RG 560 \\
\hline HTU & & $\mathrm{BA}$ & RG 331 \\
\hline HTU & BMM & & RG 302 \\
\hline HTU & BMM & & RG 414 \\
\hline
\end{tabular}


Apéndice 1. Continuación

\begin{tabular}{|c|c|c|c|c|}
\hline TAXA & FB & & & C \\
\hline Hexalectris grandiflora (A.Rich. \& Galeotti) L.O.Williams & HTS & & $\mathrm{BA}$ & RG 265, 347 \\
\hline Homalopetalum pachyphyllum (L.O.Williams) Dressler* & HEU & BMM & & RG 458 \\
\hline Laelia autumnalis fo. xanthotrophis (Rchb.f.) Halb. \& Soto Arenas & HEU & BMM & & RG 466 \\
\hline Malaxis brachyrrhynchos (Rchb.f.) Ames & HTU & BMM & $\mathrm{BA}$ & RG 394 \\
\hline Malaxis brachystachya (Lindl.) Rchb.f. & HTU & BMM & $\mathrm{BA}$ & RG 358 \\
\hline Malaxis unifolia Michx. & HTU & BMM & & RG 373 \\
\hline Oestlundorchis eriophora (Rob. \& Greenm.) Szlach. & HTU & BMM & $\mathrm{BA}$ & RG 239 \\
\hline Oncidium graminifolium (Lindl.) Lindl. & $H T(R) U$ & BMM & & RG 242 \\
\hline Platanthera brevifolia (Greene) Kraenzl. & HTU & BMM & & RG 388 \\
\hline Pleurothallis resupinata Ames** & HEU & BMM & & RG 559 \\
\hline $\begin{array}{l}\text { Stelis villosa (Knowles \& Westc.) Pridgeon \& M.W.Chase } \\
\text { POACEAE }\end{array}$ & $\mathrm{HEU}$ & BMM & & RG 457 \\
\hline Avena sativa $\mathrm{L}$. & HTU & & $\mathrm{BA}$ & RG 431 \\
\hline Festuca breviglumis Swallen & HTU & & $\mathrm{BA}$ & RG 557 \\
\hline Muhlenbergia macroura (Kunth) Hitchc. & HTU & & $\mathrm{BA}$ & RG 535 \\
\hline Oplismenus burmanii (Retz.) P.Beauv. var. burmanii & HTU & BMM & $\mathrm{BA}$ & RG 225 \\
\hline Panicum bulbosum Kunth & HTU & & $\mathrm{BA}$ & RG 397 \\
\hline Piptochaetium virescens (Kunth) Parodi & HTU & & $\mathrm{BA}$ & RG 400 \\
\hline Sporobolus indicus (L.) R.Br. & HTU & BMM & & RG 477 \\
\hline $\begin{array}{l}\text { Zeugites americanus var. pringlei (Scribn.) McVaugh } \\
\text { SMILACACEAE }\end{array}$ & HTU & BMM & BA & RG 492 \\
\hline Smilax moranensis M.Martens \& Galeotti & BTU & BMM & $\mathrm{BA}$ & RG 463 \\
\hline \multicolumn{5}{|l|}{ Eudicotiledóneas } \\
\hline \multicolumn{5}{|l|}{ ACANTHACEAE } \\
\hline Dyschoriste mcvaughii T.F.Daniel** & HTU & BMM & & RG 233 \\
\hline $\begin{array}{l}\text { Pseuderanthemum praecox (Benth.) Leonard } \\
\text { ANACARDIACEAE }\end{array}$ & HTU & BMM & & RG 234 \\
\hline $\begin{array}{l}\text { Toxicodendron radicans subsp. barkleyi Gillis } \\
\text { APIACEAE }\end{array}$ & BTU & BMM & $\mathrm{BA}$ & RG 76 \\
\hline Donnellsmithia mexicana (B.L.Rob.) Mathias \& Constance* & HTU & BMM & $\mathrm{BA}$ & RG 330, RG 450 \\
\hline $\begin{array}{l}\text { Donnellsmithia juncea (Humb. \& Bonpl. ex Spreng.) } \\
\text { Mathias \& Constance }\end{array}$ & HTU & BMM & $\mathrm{BA}$ & RG 438, 490 \\
\hline Donnellsmithia sp. & HTU & BMM & & RG 407 \\
\hline Eryngium mexiae Constance* & HTU & & $\mathrm{BA}$ & RG 228 \\
\hline Neogoezia planipetala (Hemsl.) Hemsl.* & HTU & & $\mathrm{BA}$ & RG 485 \\
\hline $\begin{array}{l}\text { Prionosciadium sp. } \\
\text { APOCYNACEAE }\end{array}$ & aTU & & $\mathrm{BA}$ & RG 646 \\
\hline Asclepias pringlei (Greenm.) Woodson* & HTU & & $\mathrm{BA}$ & RG 321 \\
\hline $\begin{array}{l}\text { Metastelma aff. latifolium Rose } \\
\text { AQUIFOLIACEAE }\end{array}$ & HTU & BMM & & RG 449 \\
\hline $\begin{array}{l}\text { Ilex tolucana Hemsl. } \\
\text { ARALIACEAE }\end{array}$ & ATU & BMM & $\mathrm{BA}$ & RG 50, 80 \\
\hline Dendropanax arboreus (L.) Decne. \& Planch. & ATU & BMM & & RG 190 \\
\hline $\begin{array}{l}\text { Oreopanax xalapensis (Kunth) Decne. \& Planch } \\
\text { ASTERACEAE }\end{array}$ & ATU & BMM & & RG 124 \\
\hline Acourtia nelsonii (B.L.Rob.) Reveal \& R.M.King** & HTU & BMM & & RG 222 \\
\hline Ageratina areolaris (DC.) Gage ex B.L.Turner & aTU & BMM & $\mathrm{BA}$ & RG 161, 533 \\
\hline Ageratina cerifera (McVaugh) R.M.King \& H.Rob. ${ }^{* *}$ & aTU & BMM & & RG 433 \\
\hline Ageratina choricephala (B.L.Rob.) R.M.King \& H.Rob.* & aTU & BMM & & RG 184 \\
\hline
\end{tabular}


Apéndice 1. Continuación

\begin{tabular}{|c|c|c|c|c|}
\hline TAXA & FB & \multicolumn{2}{|c|}{ TV } & C \\
\hline Ageratina dolichobasis (McVaugh) R.M.King \& H.Rob.* & HTU & BMM & & RG 163 \\
\hline Ageratina mairetiana (DC.) R.M.King \& H.Rob. & aTU & BMM & $\mathrm{BA}$ & RG 144 \\
\hline Baccharis heterophylla Kunth & aTU & & $\mathrm{BA}$ & RG 255 \\
\hline Barkleyanthus salicifolius (Kunth) H.Rob. \& Brettell & aTU & BMM & & RG 245 \\
\hline Bidens chiapensis var. feddemana (Sherff) Melchert & HTU & BMM & & JG 810; RG 277 \\
\hline Bidens odorata Cav. & HTU & & $\mathrm{BA}$ & RG 539 \\
\hline Brickellia squarrosa aff. var. oligodena B.L.Rob.* & aTU & & $\mathrm{BA}$ & RG 212 \\
\hline Chaptalia leucocephala Greene* & HTU & BMM & & RG 514 \\
\hline Chromolaena ovaliflora (Hook. \& Arn.) R.M.King \& H.Rob* & aTU & BMM & & RG 195 \\
\hline Cirsium ehrenbergii Sch.Bip.* & HTU & BMM & $\mathrm{BA}$ & RG 230 \\
\hline Cosmos bipinnatus Cav. & HTU & BMM & $\mathrm{BA}$ & RG 143 \\
\hline Cosmos carvifolius Benth.* & HTU & BMM & $\mathrm{BA}$ & AC 2468; RG 476 \\
\hline Cosmos stellatus Sherff** & HTU & BMM & $\mathrm{BA}$ & AC 2501; RG 426 \\
\hline Dahlia coccinea Cav. & HTU & BMM & & $\begin{array}{l}\text { AC } 2470,2502 ; \\
\text { RG } 389\end{array}$ \\
\hline Erigeron delphinifolius Willd. & HTU & & $\mathrm{BA}$ & RG 503 \\
\hline Galinsoga parviflora Cav. & HTU & BMM & $\mathrm{BA}$ & RG 479 \\
\hline Gnaphalium americanum Mill. & HTU & & $\mathrm{BA}$ & RG 275, 345 \\
\hline Heterotheca inuloides var. rosei B.Wagenkn.* & HTU & & $\mathrm{BA}$ & RG 380 \\
\hline Hieracium aff. fendleri Sch.Bip. & HTU & BMM & & RG 420 \\
\hline Hieracium pringlei A.Gray & HTU & BMM & & RG 425 \\
\hline lostephane heterophylla (Cav.) Benth.* & HTU & BMM & $\mathrm{BA}$ & RG 412 \\
\hline Jaegeria hirta (Lag.) Less & HTU & & $\mathrm{BA}$ & RG 506 \\
\hline Melampodium divaricatum (Rich.) DC. & HTU & & $\mathrm{BA}$ & RG 509 \\
\hline Melampodium perfoliatum (Cav.) Kunth & HTU & BMM & & RG 362 \\
\hline Pinaropappus roseus (Less.) Less. var. roseus & HTU & & $\mathrm{BA}$ & RG 353 \\
\hline Podachaenium eminens (Lag.) Sch.Bip. & ATU & & $\mathrm{BA}$ & RG 328 \\
\hline Psacalium eriocarpum (Hand.-Mazz.) S.F.Blake** & HTU & BMM & $\mathrm{BA}$ & RG 160 \\
\hline Roldana chapalensis (S.Watson) H.Rob. \& Brettell* & aTU & BMM & & RG 92 \\
\hline Roldana hartwegii (Benth.) H.Rob. \& Brettell & $H T(R) U$ & BMM & BA & RG 551 \\
\hline Roldana michoacana (B.L.Rob.) H.Rob. \& Brettell* & HTU & BMM & & RG 459 \\
\hline Roldana sessilifolia (Hook. \& Arn.) H.Rob. \& Brettell* & HTU & BMM & & RG 552 \\
\hline Rumfordia floribunda DC.* & $\mathrm{aRU}$ & & $\mathrm{BA}$ & RG 192 \\
\hline Stevia monardifolia Kunth* & HTU & BMM & $\mathrm{BA}$ & RG 134, 553 \\
\hline Tagetes foetidissima DC. & HTU & & BA & RG 139 \\
\hline Tagetes micrantha Cav. & HTU & & $\mathrm{BA}$ & RG 508 \\
\hline Telanthophora jaliscana H.Rob \& Brettell* & ATU & BMM & $\mathrm{BA}$ & RG 100 \\
\hline Verbesina oncophora var. subhumata McVaugh* & $\mathrm{aRU}$ & BMM & & RG 135 \\
\hline Verbesina sp. & $\mathrm{aRU}$ & BMM & & RG 158 \\
\hline Vernonia bealliae McVaugh* & $\mathrm{aRU}$ & BMM & $\mathrm{BA}$ & RG 220 \\
\hline Viguiera cordata var. websteri (B.L.Turner) McVaugh* & $\mathrm{aRU}$ & & $\mathrm{BA}$ & RG 486 \\
\hline Viguiera ensifolia (Sch.Bip.) S.F.Blake* & $\mathrm{aRU}$ & BMM & & RG 181 \\
\hline Zinnia angustifolia Kunth var. angustifolia* & HTU & BMM & $\mathrm{BA}$ & RG 140, 429 \\
\hline BEGONIACEAE & & & & \\
\hline Begonia gracilis Kunth & HTU & BMM & & RG 390 \\
\hline Begonia tapatia Burt-Utley \& McVaugh & HTU & BMM & & RG 309 \\
\hline BETULACEAE & & & & \\
\hline Alnus jorullensis Kunth subsp. jorullensis & ATU & BMM & $\mathrm{BA}$ & RG 61, 191 \\
\hline Ostrya virginiana (Mill.) K.Koch & ATU & BMM & & RG 257 \\
\hline
\end{tabular}


Apéndice 1. Continuación

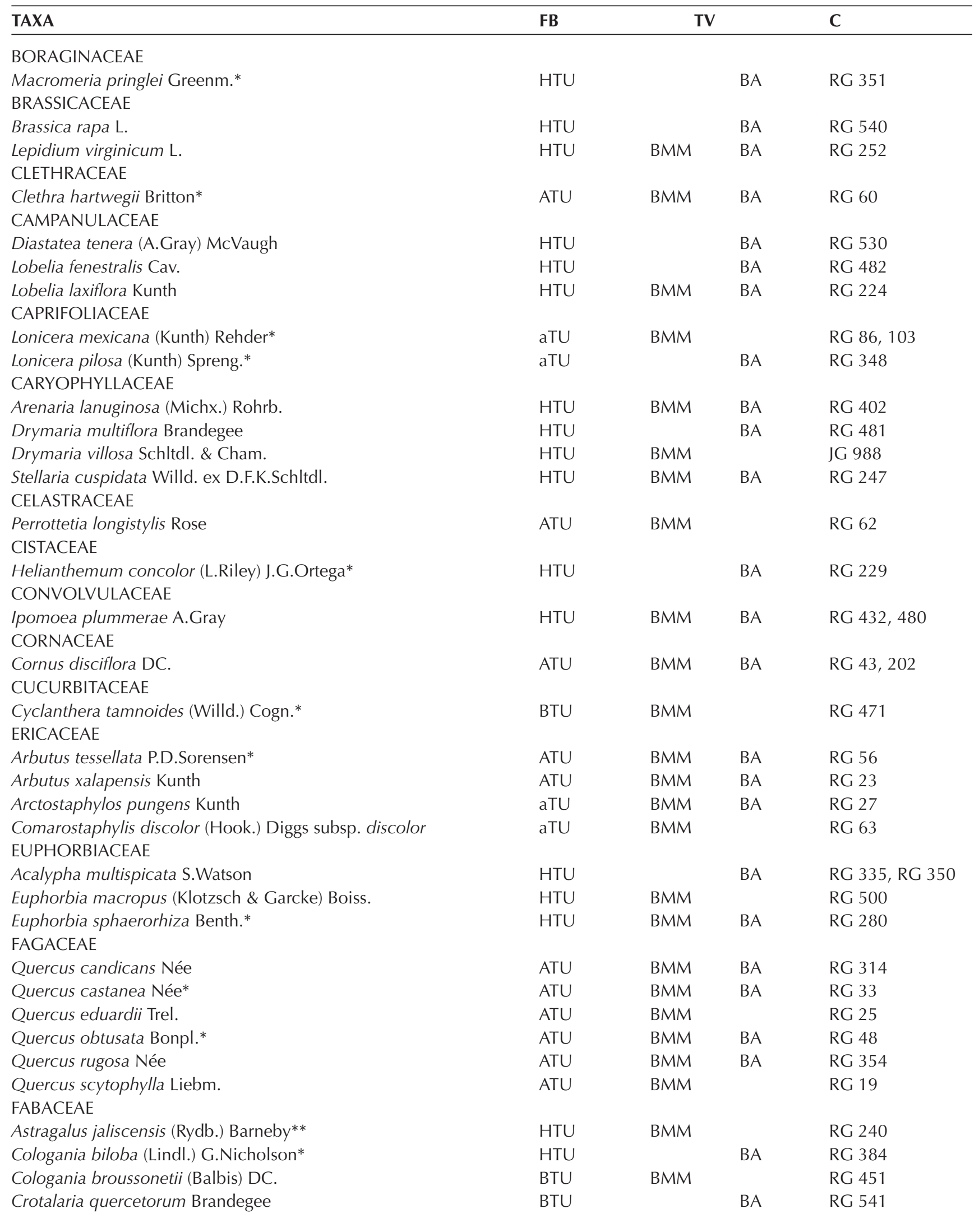


Apéndice 1. Continuación

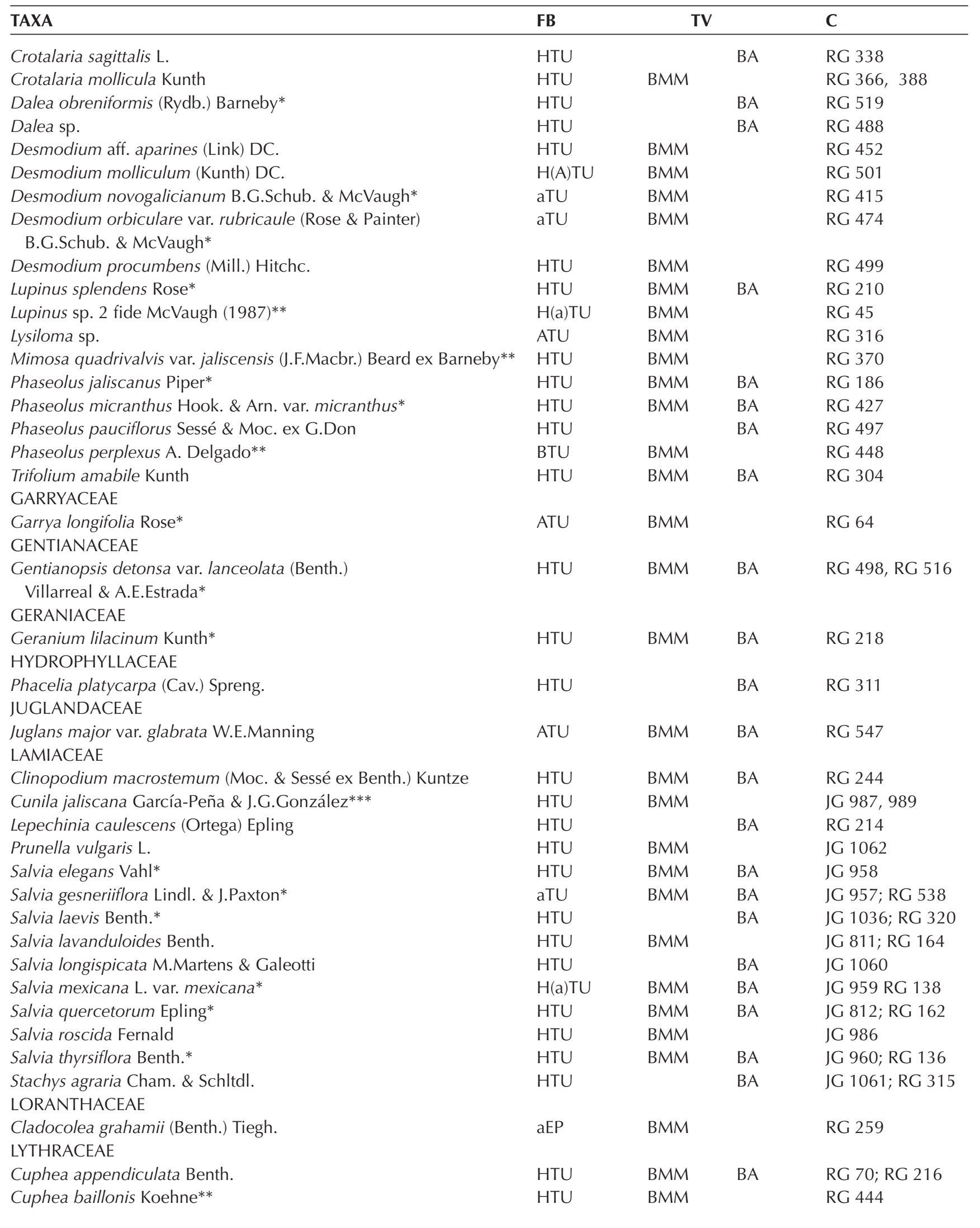


Apéndice 1. Continuación

\begin{tabular}{|c|c|c|c|c|}
\hline \multirow{2}{*}{$\begin{array}{l}\text { TAXA } \\
\text { Cuphea jorullensis Kunth* }\end{array}$} & \multirow{2}{*}{$\begin{array}{l}\text { FB } \\
\text { HTU }\end{array}$} & \multicolumn{2}{|c|}{ TV } & \multirow{2}{*}{$\begin{array}{l}\text { C } \\
\text { RG } 291\end{array}$} \\
\hline & & BMM & $\mathrm{BA}$ & \\
\hline Cuphea hookeriana Walp. & HTU & BMM & $\mathrm{BA}$ & RG 182 \\
\hline Cuphea watsoniana Koehne & HTU & BMM & & RR 775 \\
\hline Cuphea wrightii A.Gray & HTU & BMM & & RG 470 \\
\hline \multicolumn{5}{|l|}{ MALVACEAE } \\
\hline Sida haenkeana C.Presl & HTU & BMM & & RG 475 \\
\hline Sida rhombifolia L. & HTU & BMM & & RG 131 \\
\hline Tilia americana var. mexicana (Schltdl.) Hardin & ATU & BMM & & RG 83 \\
\hline Triumfetta columnaris Hochr.* & aTU & BMM & $\mathrm{BA}$ & RG 349 \\
\hline $\begin{array}{l}\text { Triumfetta semitriloba Jacq. } \\
\text { OLEACEAE }\end{array}$ & aTU & BMM & & RG 511 \\
\hline $\begin{array}{l}\text { Fraxinus uhdei (Wenz.) Lingelsh. } \\
\text { ONAGRACEAE }\end{array}$ & ATU & BMM & $\mathrm{BA}$ & RG 94 \\
\hline Fuchsia obconica Breedlove & aTU & BMM & $\mathrm{BA}$ & RG 157, 213 \\
\hline Lopezia miniata Lag. ex DC. & HTU & BMM & $\mathrm{BA}$ & RG 507 \\
\hline Lopezia racemosa Cav. & HTU & & $\mathrm{BA}$ & RG 505 \\
\hline \multicolumn{5}{|l|}{ OXALIDACEAE } \\
\hline Oxalis corniculata L. & HTU & BMM & $\mathrm{BA}$ & RG 289 \\
\hline $\begin{array}{l}\text { Oxalis hernandezii DC.* } \\
\text { PAPAVERACEAE }\end{array}$ & HTU & BMM & $\mathrm{BA}$ & RG 264 \\
\hline $\begin{array}{l}\text { Argemone ochroleuca Sweet subsp. ochroleuca* } \\
\text { PASSIFLORACEAE }\end{array}$ & \multicolumn{3}{|c|}{ PASSIFLORACEAE } & RG 303 \\
\hline \multicolumn{5}{|l|}{ PENTAPHYLACACEAE } \\
\hline Cleyera integrifolia (Benth.) Choisy* & ATU & BMM & & RG $13,35,46$ \\
\hline \multicolumn{5}{|l|}{ PLANTAGINACEAE } \\
\hline Mecardonia procumbens (Mill.) Small & HTU & & $\mathrm{BA}$ & RG 344 \\
\hline Penstemon miniatus subsp. apateticus (Straw) Straw* & HTU & BMM & $\mathrm{BA}$ & RG 478 \\
\hline Plantago australis Lam. & HTU & & $\mathrm{BA}$ & RG 141 \\
\hline Plantago nivea Kunth & HTU & BMM & & RG 375 \\
\hline \multicolumn{4}{|l|}{ PHYTOLACCACEAE } & RG 464 \\
\hline \multicolumn{5}{|l|}{ POLYGALACEAE } \\
\hline Monnina schlechtendaliana D.Dietr. & HTU & BMM & & RG 73 \\
\hline Monnina xalapensis Kunth & aTU & BMM & $\mathrm{BA}$ & RG 215 \\
\hline \multicolumn{4}{|l|}{ POLYGONACEAE } & RG 266 \\
\hline $\begin{array}{l}\text { Rumex obtusifolius L. } \\
\text { PRIMULACEAE }\end{array}$ & HTU & BMM & $\mathrm{BA}$ & RG 251 \\
\hline Anagallis arvensis L. & HTU & & $\mathrm{BA}$ & RG 562 \\
\hline $\begin{array}{l}\text { Myrsine juergensenii (Mez) Ricketson \& Pipoly } \\
\text { RANUNCULACEAE }\end{array}$ & ATU & BMM & & RG 263 \\
\hline Ranunculus mexiae (L.D.Benson) T.Duncan* & HTU & & $\mathrm{BA}$ & RG 343 \\
\hline Thalictrum gibbosum Lecoy.* & HTU & BMM & $\mathrm{BA}$ & RG 418 \\
\hline Thalictrum pringlei S.Watson* & HTU & BMM & & RG 392 \\
\hline RHAMNACEAE & & & & \\
\hline Rhamnus hintonii M.C.Johnst. \& L.A.Johnst.* & $\mathrm{A}(\mathrm{a}) \mathrm{TU}$ & BMM & & RG 194 \\
\hline
\end{tabular}


Apéndice 1. Continuación

\begin{tabular}{|c|c|c|c|c|}
\hline \multirow{2}{*}{ ROSACEAE } & \multirow[t]{2}{*}{ FB } & \multicolumn{2}{|c|}{ TV } & \multirow[t]{2}{*}{$\mathrm{C}$} \\
\hline & & & & \\
\hline Alchemilla pringlei (Rydb.) Fedde & HTU & BMM & $\mathrm{BA}$ & RG 396 \\
\hline Crataegus pubescens (C.Presl) C.Presl & ATU & & $\mathrm{BA}$ & RG 30 \\
\hline Prunus serotina subsp. capuli (Cav.) McVaugh & ATU & BMM & $\mathrm{BA}$ & RG 223 \\
\hline $\begin{array}{l}\text { Rubus schiedeanus Steud. } \\
\text { RUBIACEAE }\end{array}$ & HTU & BMM & $\mathrm{BA}$ & RG 283 \\
\hline Bouvardia rosea Schltdl. & HTU & BMM & & RG 517 \\
\hline Chiococca phaenostemon Schltdl. **** & ATU & BMM & & RG 496 \\
\hline Crusea longiflora (Roem. ex Schult.) W.R.Anderson & HTU & BMM & $\mathrm{BA}$ & RG 510 \\
\hline Crusea psyllioides (Kunth) W.R.Anderson & HTU & BMM & $\mathrm{BA}$ & RG 502 \\
\hline $\begin{array}{l}\text { Galium mexicanum Kunth subsp. mexicanum } \\
\text { SABIACEAE }\end{array}$ & BTU & & $\mathrm{BA}$ & RG 273 \\
\hline $\begin{array}{l}\text { Meliosma dentata (Liebm.) Urb. } \\
\text { SALICACEAE }\end{array}$ & ATU & BMM & & RG 34, 58 \\
\hline Salix jaliscana M.E.Jones** & ATU & BMM & & RG 196 \\
\hline $\begin{array}{l}\text { Salix aff. humboldtiana Willd. } \\
\text { SANTALACEAE }\end{array}$ & ATU & BMM & & RG 254 \\
\hline $\begin{array}{l}\text { Arceuthobium globosum Hawksw. \& Wiens } \\
\text { SCROPHULARIACEAE }\end{array}$ & $\mathrm{aEP}$ & BMM & $\mathrm{BA}$ & RG 236 \\
\hline $\begin{array}{l}\text { Buddleja sessiliflora Kunth } \\
\text { SOLANACEAE }\end{array}$ & aTU & & $\mathrm{BA}$ & RG 226 \\
\hline Cestrum confertiflorum Schltdl. & aTU & & $\mathrm{BA}$ & RG 534 \\
\hline Cestrum terminale Dunal & aTU & BMM & $\mathrm{BA}$ & RG 132 \\
\hline Jaltomata procumbens (Cav.) J.L.Gentry & HTU & BMM & $\mathrm{BA}$ & RG 428, 504 \\
\hline Lycianthes mociniana (Dunal) Bitter & HTU & BMM & $\mathrm{BA}$ & RG 325,383 \\
\hline Physalis orizabae Dunal & HTU & BMM & $\mathrm{BA}$ & RG 329,336 \\
\hline Solanum appendiculatum Dunal & BTU & BMM & & RG 462 \\
\hline Solanum brachystachys Dunal & aTU & BMM & $\mathrm{BA}$ & RG 69 \\
\hline Solanum hougasii Correll & $\mathrm{H}(\mathrm{a}) \mathrm{TU}$ & & $\mathrm{BA}$ & RG 387 \\
\hline Solanum lanceolatum Cav. & aTU & & $\mathrm{BA}$ & RG 317 \\
\hline Solanum nigrescens M.Martens \& Galeotti & HTU & & $\mathrm{BA}$ & RG 323 \\
\hline $\begin{array}{l}\text { Solanum stoloniferum Schltdl. \& Bouché* } \\
\text { STYRACACEAE }\end{array}$ & HTU & & $\mathrm{BA}$ & RG 341 \\
\hline $\begin{array}{l}\text { Styrax ramirezii Greenm. } \\
\text { SYMPLOCACEAE }\end{array}$ & ATU & BMM & $\mathrm{BA}$ & RG 37, 55 \\
\hline $\begin{array}{l}\text { Symplocos citrea Lex. ex Llave \& Lex.* } \\
\text { VALERIANACEAE }\end{array}$ & ATU & BMM & $\mathrm{BA}$ & RG 204, 279 \\
\hline $\begin{array}{l}\text { Valeriana urticifolia Kunth var. urticifolia } \\
\text { VERBENACEAE }\end{array}$ & HTU & BMM & $\mathrm{BA}$ & RG 491 \\
\hline Verbena carolina L. & HTU & BMM & $\mathrm{BA}$ & RG 89 \\
\hline $\begin{array}{l}\text { Lippia umbellata Cav. } \\
\text { VIOLACEAE }\end{array}$ & ATU & BMM & & RG 199 \\
\hline $\begin{array}{l}\text { Viola grahamii Benth. } \\
\text { VITACEAE }\end{array}$ & HTU & BMM & $\mathrm{BA}$ & RG 290 \\
\hline Vitis bourgaeana Planch. & BTU & BMM & & RG 313 \\
\hline Vitis tiliifolia Humb. \& Bonpl. ex Schult. & BTU & BMM & & RG 243 \\
\hline
\end{tabular}

